\title{
Three-dimensional reaction-convection-diffusion analysis with temperature influence for biodiesel synthesis in micro-reactors
}

\author{
Péricles C. Pontes ${ }^{\mathrm{a}, \mathrm{b}}$, Carolina P. Naveira-Cotta ${ }^{\mathrm{a}, ~}{ }^{*}$, João N.N. Quaresma ${ }^{\mathrm{c}}$ \\ ${ }^{a}$ Laboratory of Nano- and Microfluidics and Microsystems, LabMEMS, Mechanical Engineering Dept. (PEM) \& Nanoengineering Dept. (PENT), Federal \\ University of Rio de Janeiro-UFRJ, Rio de Janeiro, RJ, Brazil \\ ${ }^{\mathrm{b}}$ Araguaia Institute of Engineering, Federal University of South and Southeast of Pará-UNIFESSPA, Campus Santana do Araguaia, Bel Recanto, Av. Albino \\ Malzoni, 234, 68560-000, Santana do Araguaia, PA, Brazil \\ ${ }^{\mathrm{c}}$ Process Simulation Laboratory, School of Chemical Engineering, Federal University of Pará, FEQ/UFPA, Campus Universitário do Guamá, Rua Augusto \\ Corrêa, 01, 66075-110, Belém, PA, Brazil
}

\section{A R T I C L E I N F O}

\section{Article history:}

Received 24 September 2016

Received in revised form

9 March 2017

Accepted 13 March 2017

Available online 28 April 2017

\section{Keywords:}

Micro-reactor

Transesterification

Biodiesel

Integral transforms

Reaction-convection-diffusion equations

Hybrid methods

\begin{abstract}
A B S T R A C T
A three-dimensional nonlinear reaction-convection-diffusion model is proposed to analyze mass transfer with temperature effects, within micro-reactors of rectangular cross-section, towards the simulation of the transesterification process in biodiesel synthesis. The coupled nonlinear partial differential equations that govern the concentration of the species involved in the synthesis, is obtained from the general three-dimensional species balance equations for an isothermal and steady state system with constant physical properties. A kinetic model is considered to describe the variation of the species due to the transesterification process, assuming second order homogeneous and reversible chemical reactions. The velocity profile is analytically derived from the Navier-Stokes equations, considering fully developed stratified laminar flow of two immiscible Newtonian fluids in a rectangular cross section micro-reactor, with a plane interface between them and without field forces, and obtained by the Classical Integral Transform Technique (CITT). The nonlinear mass transport equations are then solved by the hybrid numerical-analytical approach known as the Generalized Integral Transform Technique (GITT). In the present GITT solution, two alternative eigenvalue problems are proposed, accounting or not accounting for the space variable velocity profile influence in the eigenfunction expansion basis, in order to investigate the differences in the convergence behavior of the solutions obtained through the two different auxiliary problems. The results for the concentration profiles of the biodiesel and triglyceride species are critically compared with literature data for the limiting case of parallel plates, showing a good agreement among them. The analysis also indicates that improved convergence rates are achievable through the eigenvalue problem with variable coefficients. The effects of governing parameters such as residence time, micro-reactor dimensions, and especially temperature, are investigated. It is then inspected the relative gains in triglycerides conversion rates at higher temperatures and residence times and lower micro-reactor hydraulic diameter.
\end{abstract}

๑) 2017 Elsevier Masson SAS. All rights reserved.

\section{Introduction}

Sustainable development premises have been pushing efforts, worldwide, in developing or at least improving energy generation systems and components. In this context, biofuels such as biodiesel have been progressively considered and adopted as an alternative fuel, mainly due to its feedstock flexibility and biodegradable

\footnotetext{
* Corresponding author

E-mail address: carolina@mecanica.coppe.ufrj.br (C.P. Naveira-Cotta).
}

characteristics [1]. Biodiesel is a renewable energy source that can fully or partially substitute the conventional diesel due to their similar properties, but presenting low emissions of carbon, sulfur, particulate matter, and unburned hydrocarbons [2].

The process most widely employed for the production of biodiesel is the transesterification of vegetable oils or animal fats with an alcohol, usually methanol or ethanol, in the presence of a catalyst within stirred tank or batch reactors. According to the literature [3-7], the main factors that influence the transesterification reaction are the residence time, the reaction temperature, the type and concentration of the catalyst, the feedstock, the solubility of the 


\begin{tabular}{|c|c|c|c|}
\hline \multicolumn{2}{|c|}{ Nomenclature } & $X, Y, Z$ & dimensionless spatial variables \\
\hline$O, S$ & transformed system coefficients & \multicolumn{2}{|c|}{ Greek symbols } \\
\hline C & dimensionless concentration & \multicolumn{2}{|c|}{$\beta, \gamma, v, \lambda$ eigenvalues } \\
\hline$C^{*}$ & dimensional concentration, mol. $\mathrm{m}^{-3}$ & \multicolumn{2}{|c|}{$\Phi, \Omega, \phi, \varphi, \Gamma, \Psi$ eigenfunctions } \\
\hline$D$ & diffusion coefficient, $\mathrm{m}^{2} \cdot \mathrm{s}^{-1}$ & $\delta$ & Kronecker delta \\
\hline$D_{h}$ & hydraulic diameter, m & $\mu$ & dynamic viscosity, Pa.s \\
\hline$E$ & activation energy, J.mol ${ }^{-1}$ & $\rho$ & density, $\mathrm{kg} \cdot \mathrm{m}^{-3}$ \\
\hline G & dimensionless reaction kinetics term & $\tau$ & residence time, $\mathrm{s}$ \\
\hline$H$ & micro-reactor height, m & & \\
\hline$k$ & kinetic constant, $\mathrm{m}^{3} \cdot \mathrm{mol}^{-1} \cdot \mathrm{s}^{-1}$ & \multicolumn{2}{|c|}{ Subscripts and superscripts } \\
\hline$L$ & micro-reactor length, m & $A$ & alcohol \\
\hline Norm & normalization integral & $A h$ & homogeneous potential for alcohol species \\
\hline MT & truncation order of algebraic system & $A v$ & average concentration \\
\hline$N T$ & number of terms in eigenfunction expansion & $B$ & biodiesel \\
\hline$P$ & pressure, Pa & $D G$ & diglyceride \\
\hline $\mathrm{Pe}$ & Péclet number & $G L$ & glycerol \\
\hline$Q$ & volumetric flow rate, $\mathrm{m}^{3} \cdot \mathrm{s}^{-1}$ & \multicolumn{2}{|c|}{$i, j, k, l, m, n$ order of eigenvalues and eigenfunctions } \\
\hline$R$ & ideal gas constant, J.mol ${ }^{-1} \cdot \mathrm{K}^{-1}$ & $M$ & methanol \\
\hline$T$ & temperature, $\mathrm{K}$ & $M G$ & monoglyceride \\
\hline$u$ & $\begin{array}{l}\text { velocity component in the longitudinal direction, } \\
\mathrm{m} \cdot \mathrm{s}^{-1}\end{array}$ & $r$ & $\begin{array}{l}\text { referred to the species involved in the } \\
\text { transesterification reaction }\end{array}$ \\
\hline$U$ & $\begin{array}{l}\text { dimensionless velocity component in the longitudinal } \\
\text { direction }\end{array}$ & $\begin{array}{l}\text { vel } \\
\text { TG }\end{array}$ & $\begin{array}{l}\text { referred to the velocity profile } \\
\text { triglyceride }\end{array}$ \\
\hline$W$ & width of rectangular micro-reactor, m & $\sim$ & normalized eigenfunction \\
\hline & hydrodynamic entry length, m & - & desired potential at the transformed domain \\
\hline$x, y, z$ & spatial variables & Aeq & equilibrium concentration of alcohol at interface \\
\hline
\end{tabular}

species reactants, the kind of alcohol and the molar ratio between triglyceride and alcohol. Due to the immiscible nature of the reactants, a considerable stirring mechanical work is required, together with high temperature levels, to favor the reaction process, which imply in unavoidable large energy input and long residence times, in the order of a few hours [8].

As an alternative to overcome the energetic cost and the large residence times, new technologies have recently emerged to enhance the synthesis of biodiesel, such as through micro-reactors, which are typical micro-devices that have been developed within the very important and recent research area called Microfluidics.

The use of micro-reactors for continuous processes with chemical reaction has received great attention due to the high flexibility and agility in obtaining results and the possibility of carrying out reactions in exceptional conditions in comparison with the traditional ones. In addition to the possibility of integration with other micro-devices, the micro-reactors allow the development of liquid-liquid, liquid-gas, catalyzed or non-catalyzed reactions under electric or magnetic field effects, with manifold introduction of reactants and with micro-channel geometries that can intensify the mixture [9-11].

The employment of micro-reactors for the reaction process, like transesterification reaction, in light of the reduced dimensions, ensured high surface area to volume ratio and shorter diffusion paths for the reactive system, which results in enhanced mass and heat transfer and faster reactions, consequently with lower energy and materials consumption [12-16]. As the two main reactants of the transesterification, triglycerides and alcohol, are immiscible fluids, a liquid-liquid two-phase flow develops in such microreactors, as the immiscible fluids are brought into contact and subjected to the same pressure gradient, with the associated properties and characteristics of the system such as interfacial tension, flow rates, viscous forces and wetting behavior of the channel walls [17]. According with Guan et al. [18,19] and Xie et al. [2], the two flow regimen that are commonly observed in microreactors during the transesterification reaction are the stratified flow, in which one phase flows on top or by the side of another, and droplet/plug flow, in which drops or plugs of one fluid are dispersed in the continuous phase represented by the other fluid [17].

Fairly recent works in the literature present promising results for the conversion of biodiesel in micro-reactors under different reactive conditions and different reactants and catalysts $[12,13,20]$. Integrated systems are also presented, suggesting the possibility of combining micro-reactors with conventional reactors [21], or even with other micro-systems [11,22], aiming at increasing the yields of biodiesel production with significant reduction on operating time. The idea of micro-chemical plant $[11,23,24]$, or of various microsystems integrated for a chemical reaction process, allows overcoming a very important disadvantage of micro-reactor, which are the low volumetric flow rates of products. Billo et al. [22], for example, presented an important paper about the design and operation of a plant for the production of biodiesel, containing various micro-reactors in a parallel association. To better understand the transesterification process in micro-reactors, for decisions in design tasks, the development of representative mathematical models to describe the physico-chemical phenomena is mandatory. Although there are well-defined transesterification reaction models for the conventional reactors $[4,25,26]$, generally such kinetic models are not suitable to describe the observed behavior at the micro-scale [27]. Therefore, it is necessary and very important to develop, first, mathematical models for mass transfer problems in micro-reactors and, second, computational tools and methodologies that provide solutions at low computational cost, to be used in both inverse problem analysis, for properties identification, and optimization analysis, for system design. 
The reaction mechanism is a very important step in the construction of a mathematical model for mass transfer in a process with chemical reaction. Many papers in the literature present a proposal for a reaction mechanism and for transesterification together with a model for the reaction rate of the species [3,4,13,20-22,27-30]. The kinetic model most used for transesterification considers a second order, consecutive and reversible reaction.

In this work, a three-dimensional nonlinear mathematical model is proposed to describe the transesterification between soybean oil and methanol in micro-reactors, catalyzed by sodium hydroxide, and considering the diffusion, reaction and convection phenomena. The set of coupled nonlinear partial differential equations is then solved by the hybrid numerical-analytical method known as the Generalized Integral Transform Technique (GITT) [31-37], which offers a robust error controlled solution within a low computational cost, by combining analytical eigenfunction expansions with the numerical solution of the ordinary differential equations for the integral transformed potentials. This hybrid method has been previously employed in the analysis of transport phenomena in reactive flows [38-42], as well as in the analysis of heat and mass transfer within microfluidic devices [37,39-41,43-48]. The numerical results for the concentration fields from the present hybrid algorithm were compared with data obtained from the literature, and a good agreement between them was verified. Here, special emphasis has been given to the analysis of the reaction temperature effects on the triglycerides conversion rates, but the effects of the volumetric flow rates, micro-reactor dimensions, residence time were also evaluated.

\section{Flow problem}

Biodiesel can be obtained from the transesterification reaction between triglycerides and alcohol (alcoholysis reaction) in the presence of a catalyst, typically sodium or potassium hydroxide, which yields esters of fatty acids and glycerol. A general mechanism widely accepted in the literature considers the transesterification as a second order reversible and homogeneous reaction, exhibiting kinetics in accordance with Eq. (1) [4,13,39-41]:

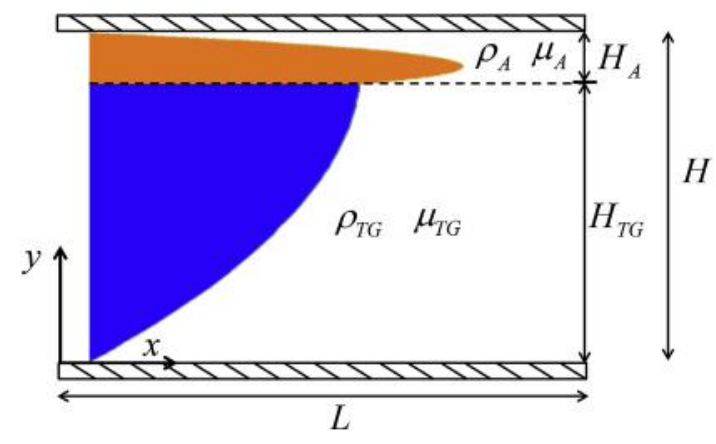

Fig. 1. Velocity profile for the fully developed stratified flow in micro-reactors.

developed stratified laminar flow of two immiscible Newtonian fluids, soybean oil and methanol, with constant physical properties in a rectangular cross section micro-reactor, subject to a constant pressure drop and under isothermal steady conditions. The body forces influence is neglected and a flat plane interface between the two fluids is considered, located at $H_{T G}$. At the interface it is assumed continuity of velocity and shear stress between the two fluid layers, while at the walls it is adopted the usual no-slip conditions. From these assumptions, the Navier-Stokes equations are simplified and can be written as:

$$
\begin{aligned}
& \frac{\partial P}{\partial x}=\mu_{T G}\left(\frac{\partial^{2} u_{T G}}{\partial y^{2}}+\frac{\partial^{2} u_{T G}}{\partial z^{2}}\right) ; \\
& \frac{\partial P}{\partial x}=\mu_{A}\left(\frac{\partial^{2} u_{A}}{\partial y^{2}}+\frac{\partial^{2} u_{A}}{\partial z^{2}}\right), \\
& 0<z<W, H_{T G}<y<H \\
& u_{T G}(y, 0)=u_{T G}(y, W)=u_{A}(y, 0)=u_{A}(y, W)=0 \\
& u_{T G}(0, z)=u_{A}(H, z)=0
\end{aligned}
$$

$$
\begin{gathered}
\text { Triglyceride }(\mathrm{TG})+\operatorname{Alcohol}(\mathrm{A}) \underset{\mathrm{k}_{2}}{\stackrel{\mathrm{k}_{1}}{\rightleftarrows}} \operatorname{Diglyceride}(\mathrm{DG})+\operatorname{Biodiesel}(\mathrm{B}) \\
\text { Diglyceride }(\mathrm{DG})+\operatorname{Alcohol}(\mathrm{A}) \underset{\mathrm{k}_{4}}{\stackrel{\mathrm{k}_{3}}{\rightleftarrows}} \text { Monoglyceride }(\mathrm{MG})+\operatorname{Biodiesel}(\mathrm{B}) \\
\text { Monoglyceride }(\mathrm{MG})+\operatorname{Alcohol}(\mathrm{A}) \underset{\mathrm{k}_{6}}{\stackrel{\mathrm{k}_{5}}{\rightleftarrows}} \text { Glycerol }(\mathrm{GL})+\operatorname{Biodiesel}(\mathrm{B})
\end{gathered}
$$

Diglycerides and monoglycerides are intermediates and considered as contaminants in the final product [25].

The reactants for the transesterification reaction, triglycerides and alcohol, present an immiscible nature and, therefore, in a continuous process inside a micro-reactor, they form a liquid-liquid two-phase flow. In this work, it will be assumed that a stratified flow pattern is established between these two reactant fluids. A schematic description of the stratified velocity profiles is shown in Fig. 1.

The mathematical model that describes the velocity profiles can be obtained from de Navier-Stokes equations, assuming fully

$$
\begin{aligned}
& u_{T G}\left(H_{T G}, z\right)=u_{A}\left(H_{T G}, z\right) \\
& -\left.\mu_{T G} \frac{\partial u_{T G}}{\partial y}\right|_{y=H_{T G}}=-\left.\mu_{A} \frac{\partial u_{A}}{\partial y}\right|_{y=H_{T G}}
\end{aligned}
$$

The pressure gradient is considered constant along the microreactor and it can be defined by [13]:

$$
\frac{\partial P}{\partial x}=-\frac{\Delta P}{L}
$$

An analytical solution for the problem defined by Eqs. (2) can be obtained through the Classical Integral Transform Technique (CITT) $[49,50]$. For this purpose, a suitable eigenvalue problem to 
represent the velocity potential for the two fluids in the $\mathrm{z}$ direction should be proposed, in order to allow for the elimination of the width coordinate, $z$, through integral transformation of Eqs. (2.a-j). After the integral transformation procedure (see Appendix A), infinite systems of second order ordinary differential equations are obtained, which are coupled at the interface $y=H_{T G}$. These systems can be solved analytically [51] and their solutions are then used to explicitly represent the velocity fields of the two fluids through the inversion formula. Therefore, the velocity profile of the two fluids, methanol and soybean oil, can be written in terms of hyperbolic functions as follows:

$$
\begin{aligned}
& \frac{Q_{T G}}{Q_{A}}= \frac{W H_{T G}\left(\frac{1}{H_{T G} W} \int_{0}^{W} \int_{0}^{H_{T G}} u_{T G}(y, z) d y d z\right)}{W\left(H-H_{T G}\right)\left(\frac{1}{\left(H-H_{T G}\right) W} \int_{0}^{W} \int_{H_{T G}}^{H} u_{A}(y, z) d y d z\right)} \\
&=\frac{\int_{0}^{W} \int_{0}^{H_{T G}} u_{T G}(y, z) d y d z}{\int_{0}^{W} \int_{H_{T G}}^{H} u_{A}(y, z) d y d z}
\end{aligned}
$$

$$
\begin{gathered}
u_{T G}(y, z)=\sum_{i=1}^{\infty} \tilde{\Psi}_{v e l, i}(z) S_{i}\left\{\left(1+\frac{\mu_{A}}{\mu_{T G}}\right)\left[-\sinh \left(\frac{H i \pi}{W}\right)+\sinh \left(\frac{i \pi(H-y)}{W}\right)\right]+\right. \\
+\left(\frac{\mu_{A}}{\mu_{T G}}-1\right)\left[\sinh \left(\frac{\left(H-2 H_{T G}\right) i \pi}{W}\right)+\sinh \left(\frac{i \pi\left(H-H_{T G}+y\right)}{W}\right)+\right. \\
\left.\left.-\sinh \left(\frac{i \pi\left(H-H_{T G}-y\right)}{W}\right)-\sinh \left(\frac{i \pi\left(H-2 H_{T G}+y\right)}{W}\right)\right]+2 \sinh \left(\frac{i \pi y}{W}\right)\right\}
\end{gathered}
$$

$$
\begin{gathered}
u_{A}(y, z)=\sum_{i=1}^{\infty} \tilde{\Psi}_{v e l, i}(z) S_{i}\left\{\left(1+\frac{\mu_{T G}}{\mu_{A}}\right)\left[-\sinh \left(\frac{H i \pi}{W}\right)+\sinh \left(\frac{i \pi y}{W}\right)\right]+\right. \\
+\left(1-\frac{\mu_{T G}}{\mu_{A}}\right)\left[\sinh \left(\frac{\left(H-2 H_{T G}\right) i \pi}{W}\right)+\sinh \left(\frac{i \pi\left(2 H_{T G}-y\right)}{W}\right)+\right. \\
\left.\left.-\sinh \left(\frac{i \pi\left(H-H_{T G}-y\right)}{W}\right)-\sinh \left(\frac{i \pi\left(H+H_{T G}-y\right)}{W}\right)\right]+2 \sinh \left(\frac{i \pi(H-y)}{W}\right)\right\}
\end{gathered}
$$

where,

$$
S_{i}=\frac{-2 \sqrt{2} W^{5 / 2} \Delta P}{i^{3} L \pi^{3}\left[\left(\mu_{T G}-\mu_{A}\right) \sinh \left(\frac{\left(H-2 H_{T G}\right) i \pi}{W}\right)+\left(\mu_{T G}+\mu_{A}\right) \sinh \left(\frac{H i \pi}{W}\right)\right]},
$$

$i=1,2,3, \ldots$

The velocity profiles defined by Eqs. (4.a-c) depend on the dynamic viscosities of the two fluids, the dimensions of the micro-reactor, $L, W$ and $H$, and the unknown parameters pressure drop, $\Delta P$, and the interface location, $H_{T G}$. The $\Delta P$ and $H_{T G}$ can eventually be obtained from the experimental observation, but for purposes of simulation, it is recommended that these unknown parameters should be calculated from a known parameter input of the problem, which in this case is the volumetric flow rate of each fluid.

The height of the triglyceride layer is then determined from the ratio of volumetric flow rates between the two fluids in the rectangular cross section of the micro-reactor geometry, in the form:
From the experimental volumetric flow rates of the two fluids and their dynamic viscosities, the triglyceride layer height may be implicitly calculated from Eq. (5), and the pressure drop can be calculated isolating $\Delta P$ in the volumetric flow rate equation for the triglyceride species.

\section{Mass transfer problem}

A three-dimensional mathematical model, that governs the concentration of the species involved in the transesterification reaction in a micro-reactor of rectangular cross section, was obtained from the general mass balance equations, assuming an isothermal and steady system with constant physical properties. It is considered that the reactive effects occur predominantly in the triglyceride phase and that only alcohol (methanol) species migrates through the interface and the other species remain confined in the triglyceride phase [13]. As the reaction occurs predominantly in the triglyceride phase, it is reasonable to assume that reactants, intermediates and products are subject to the same convective effects that the triglyceride phase. At the walls of the micro-reactor, it is assumed no penetrability conditions. At the inlet of the microreactor, all species have known concentration, while at the microreactor outlet it is assumed a zero flux condition for all species. 
Therefore, the dimensionless reaction-convection-diffusion equations that govern the concentration of the species in the triglyceride phase, with the specific nonlinear chemical kinetics terms, can be written as:

$U_{T G}(Y, Z) \frac{\partial C_{r}}{\partial X}=\frac{1}{P e_{r}} \frac{\partial^{2} C_{r}}{\partial X^{2}}+\xi_{r}\left(\frac{\partial^{2} C_{r}}{\partial Y^{2}}+\omega \frac{\partial^{2} C_{r}}{\partial Z^{2}}\right)+\varsigma G_{r}$,

where $r=T G, D G, M G, B, A, G L$

$C_{T G}(0, Y, Z)=1$

$C_{r}(0, Y, Z)=0$,

where $r=D G, M G, B, A, G L$

$\left.\frac{\partial C_{r}}{\partial X}\right|_{X=1}=\left.\frac{\partial C_{r}}{\partial Y}\right|_{Y=0}=\left.\frac{\partial C_{r}}{\partial Z}\right|_{Z=0}=\left.\frac{\partial C_{r}}{\partial Z}\right|_{Z=1}=0$,

where $r=T G, D G, M G, B, A, G L$

$C_{A}(X, 1, Z)=C_{\text {Aeq }} ;\left.\frac{\partial C_{r}}{\partial Y}\right|_{Y=1}=0$,

where $r=T G, D G, M G, B, G L$

where $G_{r}$ represents the reaction kinetic term for each species involved in the transesterification. Considering a second-order consecutive reversible reaction $[4,13,39,40]$, the reaction kinetic terms for the species involved in the transesterification can be written according to Table 1.

The dimensionless groups used in Eq. (6) were defined as:
The GITT methodology consists of the following steps: 1) choosing an auxiliary eigenvalue problem based on the original problem; 2) determination of eigenfunctions, eigenvalues and norms; 3) development of the integral transform pair; 4) integral transformation of the original partial differential equations, resulting in an infinite and coupled ordinary differential equations system; 5) solution of the transformed ODE system and; 6) obtaining the desired potential by recalling the inverse formula $[33,37]$.

To improve the computational performance and convergence behavior in the eigenfunction expansion, and to avoid anomalous behavior, it is recommended to remove the nonhomogeneous terms in the boundaries conditions in the directions that will be transformed, since they are not accounted for by the auxiliary problem [35,37]. In this case, the only nonhomogeneous boundary condition of the alcohol species at $Y=1$ will be homogenized through the following simple filter:

$C_{A}(X, Y, Z)=C_{A h}(X, Y, Z)+C_{A e q}$

An important step in the GITT solution procedure is the choice of the auxiliary problem, for obtaining the eigenfunctions and eigenvalues utilized in the construction of the species concentrations as orthogonal eigenfunctions expansions. An eigenvalue problem that incorporates more information of the physical problem must be more representative of the process than an eigenvalue problem containing purely diffusive effects of the problem. Thus, in this paper, two alternatives for the eigenvalue problem formulation will be considered, one including the velocity profile as a space variable coefficient, and the other simpler one without the velocity field,

$C_{T G}=\frac{C_{T G}^{*}}{C_{T G o}^{*}} ; \quad C_{A}=\frac{C_{A}^{*}}{C_{T G o}^{*}} ; \quad C_{D G}=\frac{C_{D G}^{*}}{C_{T G o}^{*}} ; \quad C_{M G}=\frac{C_{M G}^{*}}{C_{T G o}^{*}} ; \quad C_{B}=\frac{C_{B}^{*}}{C_{T G o}^{*}} ; \quad C_{G L}=\frac{C_{G L}^{*}}{C_{T G o}^{*}} ; \quad C_{A e q}=\frac{C_{A e q}^{*}}{C_{T G o}} ;$

$X=\frac{x}{L} ; \quad Y=\frac{y}{H_{T G}} ; \quad Z=\frac{z}{W} ; \quad P e_{r}=\frac{u_{A v} L}{D_{r}} ; \quad U_{T G}=\frac{u_{T G}}{u_{A v}} ; \quad s=\frac{L C_{T G o}}{u_{A v}} ; \quad \xi_{r}=\frac{L D_{r}}{u_{A v} H_{T G}^{2}} ; \quad \omega=\frac{H_{T G}^{2}}{W^{2}}$

where $C_{A e q}^{*}$ is the equilibrium concentration of the alcohol species at the interface and $u_{A v}$ is the average velocity of the triglyceride phase.

Equations (6) form a system of nonlinear partial differential equations, coupled due to the kinetic terms, that can be solved by the Generalized Integral Transform Technique, GITT [31-48]. The GITT is a hybrid numerical-analytical technique derived from the Classical Integral Transform Technique, CITT [49,50] that allows obtaining solutions more accurately and at a much lower computational cost, but with more analytical involvement than conventional numerical methods. The GITT has been successfully applied to various classes of nonlinear convection-diffusion problems and it is fully documented in different sources [31-48,52].

Table 1

Dimensionless kinetic expressions for the species $r$.

\begin{tabular}{ll}
\hline Specie $\boldsymbol{r}$ & $\boldsymbol{G}_{\boldsymbol{r}}$ \\
\hline$T G$ & $-k_{1} C_{T G} C_{A}+k_{2} C_{D G} C_{B}$ \\
$A$ & $\left(-k_{1} C_{T G}-k_{3} C_{D G}-k_{5} C_{M G}\right) C_{A}+\left(k_{2} C_{D G}+k_{4} C_{M G}+k_{6} C_{G L}\right) C_{B}$ \\
$D G$ & $\left(k_{1} C_{T G}-k_{3} C_{D G}\right) C_{A}+\left(-k_{2} C_{D G}+k_{4} C_{M G}\right) C_{B}$ \\
$M G$ & $\left(k_{3} C_{D G}-k_{5} C_{M G}\right) C_{A}+\left(-k_{4} C_{M G}+k_{6} C_{G L}\right) C_{B}$ \\
$G L$ & $k_{5} C_{M G} C_{A}-k_{6} C_{G L} C_{B}$ \\
$B$ & $\left(k_{1} C_{T G}+k_{3} C_{D G}+k_{5} C_{M G}\right) C_{A}+\left(-k_{2} C_{D G}-k_{4} C_{M G}-k_{6} C_{G L}\right) C_{B}$ \\
\hline
\end{tabular}

and the convergence of the results obtained through these two approaches will be critically evaluated.

The eigenvalue problem with constant coefficients has analytical solution by the method of separation of variables. The other one with velocity profile does not have, and, in this case, the GITT will be used to reduce the differential equation in an algebraic system. After the solution of the eigenvalue problem and from the orthogonality property and norm of the eigenfunctions, it is possible to define the integral transform pair that will allow the integral transformation of Eqs. (6). The solution methodology for the two eigenvalue problems are given in Appendices B and C.

After the integral transformation procedure of Eqs. (6) is performed (see Appendices B and C), an infinite system of nonlinear coupled ordinary differential equations for the transformed concentrations of the species in the transesterification reaction is obtained, which needs to be numerically solved to provide the solution of the transformed concentration field for the species. The built in function NDSolve of the Mathematica system can be employed for this purpose [51], providing continuous solutions with automatic error control, and returned in the form of interpolation objects along the $X$ coordinate.

After obtaining the solution for the transformed system, the concentration fields of all species can then be written for the case of 
simpler eigenvalue problem as:

$$
\begin{aligned}
& C_{A h}(X, Y, Z)=\sum_{i=1}^{\infty} \tilde{\Phi}_{i}(Y, Z) \bar{C}_{A h_{i}}(X) ; \\
& C_{r}(X, Y, Z)=\sum_{i=1}^{\infty} \widetilde{\Omega}_{i}(Y, Z) \bar{C}_{r_{i}}(X)
\end{aligned}
$$

and for the case of eigenvalue problem with velocity profile:

$$
\begin{aligned}
& C_{A h}(X, Y, Z)=\sum_{i=1}^{\infty} \tilde{\Gamma}_{i}(Y, Z) \bar{C}_{A h_{i}}(X) ; \\
& C_{r}(X, Y, Z)=\sum_{i=1}^{\infty} \tilde{\Psi}_{i}(Y, Z) \bar{C}_{r_{i}}(X)
\end{aligned}
$$

where $r=T G, D G, M G, B, G . \bar{C}$ represents the transformed concentration field.

Although the reaction system is considered to be isothermal, the transesterification reaction can be evaluated at different temperature levels, since this parameter is related to the energy state of the molecules. It is expected that higher temperatures provide better triglyceride conversion rates. The Arrhenius equation can be used to represent the relation between the kinetic constant and the temperature [53]:

$k_{i, 1}=\alpha_{i} \operatorname{Exp}\left[-\frac{E_{i}}{R T_{1}}\right], \quad i=1,2, \ldots, 6$

where $k_{i, 1}$ is the kinetic constant of index $i$ at the temperature $T_{1}$. From the Arrhenius equation, it is possible to obtain an equation that relates any kinetic constant at different temperatures, independent of the pre-exponential factor. This equation can be written as:

$\ln \left(\frac{k_{i, 2}}{k_{i, 1}}\right)=\frac{E_{i}}{R}\left[\frac{1}{T_{1}}-\frac{1}{T_{2}}\right], \quad i=1,2, \ldots, 6$

\begin{tabular}{|c|c|c|c|}
\hline Parameter & Value & Parameter & Value \\
\hline $\begin{array}{l}\mu_{T G} \\
\mu_{A} \\
D_{T G} \\
D_{A} \\
D_{D G}, D_{M G}, D_{G L} \text { and } D_{B}\end{array}$ & $\begin{array}{l}5.825 \times 10^{-2} \mathrm{~Pa} \mathrm{~s} \\
5.470 \times 10^{-4} \mathrm{~Pa} \mathrm{~s} \\
1.580 \times 10^{-9} \mathrm{~m}^{2} \mathrm{~s}^{-1} \\
1.182 \times 10^{-10} \mathrm{~m}^{2} \mathrm{~s}^{-1} \\
1.380 \times 10^{-9} \mathrm{~m}^{2} \mathrm{~s}^{-1}\end{array}$ & $\begin{array}{l}k_{2} \\
k_{3} \\
k_{4} \\
k_{5} \\
k_{6}\end{array}$ & $\begin{array}{l}9.623 \times 10^{-6} \mathrm{~m}^{3} \mathrm{~mol}^{-1} \mathrm{~s}^{-1} \\
1.88 \times 10^{-5} \mathrm{~m}^{3} \mathrm{~mol}^{-1} \mathrm{~s}^{-1} \\
1.074 \times 10^{-4} \mathrm{~m}^{3} \mathrm{~mol}^{-1} \mathrm{~s}^{-1} \\
2.117 \times 10^{-5} \mathrm{~m}^{3} \mathrm{~mol}^{-1} \mathrm{~s}^{-1} \\
9.0 \times 10^{-7} \mathrm{~m}^{3} \mathrm{~mol}^{-1} \mathrm{~s}^{-1}\end{array}$ \\
\hline$\rho_{T G}$ & $885 \mathrm{~kg} \mathrm{~m}^{-3}$ & Reaction & Activation Energy \\
\hline $\begin{array}{l}Q_{T G} / Q_{A} \\
C_{T G o} \\
C_{\text {Aeq }} \\
L \\
R \\
k_{1}\end{array}$ & $\begin{array}{l}3.402 \\
1014 \mathrm{~mol} \mathrm{~m}^{-3} \\
4.4 \\
233 \times 10^{-4} \mathrm{~m} \\
8.314 \mathrm{~J} \mathrm{~mol}^{-1} \mathrm{~K}^{-1} \\
4.368 \times 10^{-6} \mathrm{~m}^{3} \mathrm{~mol}^{-1} \mathrm{~s}^{-1}\end{array}$ & $\begin{array}{l}T G \rightarrow D G \\
D G \rightarrow T G \\
D G \rightarrow M G \\
M G \rightarrow D G \\
M G \rightarrow G L \\
G L \rightarrow M G\end{array}$ & $\begin{array}{l}55.0355 \mathrm{~J} \mathrm{~mol}^{-1} \\
41.5833 \mathrm{~J} \mathrm{~mol}^{-1} \\
83.1498 \mathrm{~J} \mathrm{~mol}^{-1} \\
61.2906 \mathrm{~J} \mathrm{~mol}^{-1} \\
26.8834 \mathrm{~J} \mathrm{~mol}^{-1} \\
40.1430 \mathrm{~J} \mathrm{~mol}^{-1}\end{array}$ \\
\hline
\end{tabular}

Table 2

Input data for simulation of a micro-reactor $[4,13,39,40]$.

\begin{tabular}{|c|c|c|c|c|}
\hline Micro-reactor height, m & $50 \times 10^{-6}$ & $100 \times 10^{-6}$ & $150 \times 10^{-6}$ & $200 \times 10^{-6}$ \\
\hline Triglyceride phase height, m & $4.2222 \times 10^{-5}$ & $8.4445 \times 10^{-5}$ & $1.2667 \times 10^{-4}$ & $1.6889 \times 10^{-4}$ \\
\hline Volume of triglyceride layer, $\mathrm{m}^{3}$ & $1.5500 \times 10^{-8}$ & $2.3000 \times 10^{-8}$ & $3.4500 \times 10^{-8}$ & $4.6000 \times 10^{-8}$ \\
\hline$\tau=30 \mathrm{~s} \rightarrow Q_{T G}, \mathrm{~m}^{3} \cdot \mathrm{s}^{-1}$ & $3.8333 \times 10^{-10}$ & $7.6667 \times 10^{-10}$ & $1.1500 \times 10^{-9}$ & $1.5333 \times 10^{-9}$ \\
\hline$\tau=60 \mathrm{~s} \rightarrow Q_{T G}, \mathrm{~m}^{3} \cdot \mathrm{s}^{-1}$ & $1.9167 \times 10^{-10}$ & $3.8333 \times 10^{-10}$ & $5.7500 \times 10^{-10}$ & $7.6667 \times 10^{-10}$ \\
\hline$\tau=180 \mathrm{~s} \rightarrow Q_{T G}, \mathrm{~m}^{3} . \mathrm{s}^{-1}$ & $6.3333 \times 10^{-11}$ & $1.2833 \times 10^{-10}$ & $1.9167 \times 10^{-10}$ & $2.5500 \times 10^{-10}$ \\
\hline$\tau=600 \mathrm{~s} \rightarrow Q_{T G}, \mathrm{~m}^{3} \cdot \mathrm{s}^{-1}$ & $1.9167 \times 10^{-11}$ & $3.8333 \times 10^{-11}$ & $5.7500 \times 10^{-11}$ & $7.6667 \times 10^{-11}$ \\
\hline
\end{tabular}

Table 3

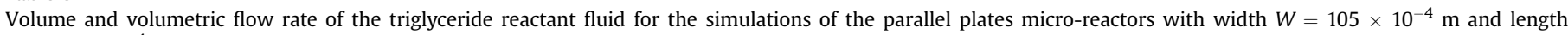
$L=233 \times 10^{-4} \mathrm{~m}$. where $k_{i, 2}$ is the kinetic constant of index $i$ at the temperature $T_{2}$.

Due to the absence of specific data on the reaction kinetics for microfluidic devices and the reaction system here proposed, using methanol and soybean oil, activation energy and pre-exponential data from a batch system were used in the present analysis to investigate the temperature effects on the process [4].

The reaction efficiency is analyzed through the fractional conversion of triglycerides for different residence times by the following equation:

Conversion $T G(\%)=\left[\frac{C_{T G, A V}(0)-C_{T G, A v}(1)}{C_{T G, A v}(0)}\right] \times 100$

The residence time provides an average time that the reactant fluids are subjected to the reactive, diffusive and convective phenomena inside the micro-reactor. It is expected that higher conversion of triglyceride will occur at higher residence times. Therefore, the residence time is calculated taking into account only the volume of the triglyceride phase, which is where the reaction takes place predominantly, according to the following equation:

$\tau=\frac{L W H_{T G}}{\mathrm{Q}_{T G}}$

\section{Results and discussion}

The results presented in this section were obtained through the subroutine NDSolve built in the Mathematica 9.0 platform in default mode [51].

The input data employed in the present study of the transesterification reaction between soybean oil and methanol were obtained from the literature $[4,13,39,40]$, and they are shown in Table 2.

Table 3 shows some values for the volume of the triglyceride layer obtained from the literature [13] for micro-reactor heights of $100 \times 10^{-6}$ and $200 \times 10^{-6} \mathrm{~m}$, as well as other values calculated for the cases of $50 \times 10^{-6}$ and $150 \times 10^{-6} \mathrm{~m}$. The triglyceride 
Table 4

Dimensionless numbers and the entrance length for the parallel plates micro-reactor.

\begin{tabular}{|c|c|c|c|c|}
\hline Micro-reactor height, m & $50 \times 10^{-6}$ & $50 \times 10^{-6}$ & $200 \times 10^{-6}$ & $200 \times 10^{-6}$ \\
\hline Triglyceride phase height, m & $4.2222 \times 10^{-5}$ & $4.2222 \times 10^{-5}$ & $1.6889 \times 10^{-4}$ & $1.6889 \times 10^{-4}$ \\
\hline$\tau, \mathrm{s}$ & 30 & 600 & 30 & 600 \\
\hline$u_{A v}, \mathrm{~m}_{\mathrm{s}} \mathrm{s}^{-1}$ & $8.6466 \times 10^{-4}$ & $4.3233 \times 10^{-5}$ & $8.6466 \times 10^{-4}$ & $4.3233 \times 10^{-5}$ \\
\hline$D_{h}, \mathrm{~m}$ & $8.4106 \times 10^{-5}$ & $8.4106 \times 10^{-5}$ & $3.3243 \times 10^{-4}$ & $3.3243 \times 10^{-4}$ \\
\hline $\operatorname{Re}$ & $1.1049 \times 10^{-3}$ & $5.5245 \times 10^{-5}$ & $4.3671 \times 10^{-3}$ & $2.1836 \times 10^{-4}$ \\
\hline$x_{e}, \mathrm{~m}$ & $5.0467 \times 10^{-5}$ & $5.0464 \times 10^{-5}$ & $1.9951 \times 10^{-4}$ & $1.9946 \times 10^{-4}$ \\
\hline$P e_{T G}$ & 12751 & 637.550 & 12751 & 637.550 \\
\hline$P e_{A}$ & 170445 & 8522.25 & 170445 & 8522.25 \\
\hline$P e_{r}, r=D G, M G, G L, B$ & 14599 & 729.949 & 14599 & 729.949 \\
\hline
\end{tabular}

volumetric flow rates can be determined from the ratio between the volume of the triglyceride layer and the desired residence time in the analysis.

The input data were also used to calculate the hydraulic diameter, average velocity, Reynolds and Péclet numbers in the triglyceride phase, and the entrance length according to the relationships given by Eqs. (15), and the results are presented in Table 4.

$$
\begin{aligned}
D_{h} & =\frac{4 W H_{T G}}{2\left(W+H_{T G}\right)} ; \\
\operatorname{Re} & =\frac{\rho_{T G} u_{A v} D_{h}}{\mu_{T G}} ; \\
\frac{x_{e}}{D_{h}} & =\frac{0.6}{1+0.035 \operatorname{Re}}+0.056 \operatorname{Re}
\end{aligned}
$$

From Table 4, it is possible to observe that for residence times and parameters adopted to simulate the velocity profile and mass transfer during the transesterification reaction in parallel plate micro-reactors, the values of the Reynolds number and the hydrodynamic entry length confirm the hypothesis of fully developed laminar flow. The Péclet values indicate a predominance of advective or convective effects in comparison to the diffusive effects in the axial direction. Thus, the axial diffusion term will not be taken into account in the simulations, then reducing the transformed boundary value problem to an initial value problem. The mass transfer simulations for the square cross section microreactors were also conducted without considering the effect of axial diffusion.

Table 5 shows some values for the volume of the triglyceride layer and its volumetric flow rate in a square cross section microreactor with height $H$ and width $W$.

Figure 2a illustrates the velocity profile in a micro-reactor with dimensions $L=233 \times 10^{-4} \mathrm{~m}, W=105 \times 10^{-4} \mathrm{~m}$ and $H=100 \times 10^{-6} \mathrm{~m}$ for $\tau=600 \mathrm{~s}$ and $Q_{T G}=2 \times 10^{-8} \mathrm{~m}^{3} \mathrm{~s}^{-1}$. For these dimension values, the hypothesis of parallel plates adopted by $\mathrm{Al}-$ Dhubabian [13] is justified since the velocity profile remains uniform along the $\mathrm{z}$ direction, being significantly affected by border effects only in the vicinity of the walls. This behavior was expected due to the high aspect ratio $(W / H)$ that is observed in this case. Figure $2 \mathrm{~b}$ illustrates the velocity profile for a square cross section micro-reactor with the dimensions $L=233 \times 10^{-4} \mathrm{~m}$ and $W=$ $H=100 \times 10^{-6} \mathrm{~m}$ for $\tau=600 \mathrm{~s}$ and $Q_{T G}=3.4667 \times 10^{-10} \mathrm{~m}^{3} \mathrm{~s}^{-1}$. In

\begin{tabular}{|c|c|c|c|c|}
\hline$H=W$ & $100 \times 10^{-6} \mathrm{~m}$ & $200 \times 10^{-6} \mathrm{~m}$ & $300 \times 10^{-6} \mathrm{~m}$ & $400 \times 10^{-6} \mathrm{~m}$ \\
\hline Triglyceride phase height, $\mathrm{m}$ & $8.9148 \times 10^{-5}$ & $1.7829 \times 10^{-4}$ & $2.6744 \times 10^{-4}$ & $3.5659 \times 10^{-4}$ \\
\hline Volume of triglyceride layer, $\mathrm{m}^{3}$ & $2.0770 \times 10^{-10}$ & $8.3080 \times 10^{-10}$ & $1.8694 \times 10^{-9}$ & $6.6469 \times 10^{-9}$ \\
\hline$\tau=30 \mathrm{~s} \rightarrow Q_{T G}, \mathrm{~m}^{3} \cdot \mathrm{s}^{-1}$ & $6.9233 \times 10^{-12}$ & $2.7695 \times 10^{-11}$ & $6.2315 \times 10^{-11}$ & $1.1078 \times 10^{-10}$ \\
\hline$\tau=60 \mathrm{~s} \rightarrow Q_{T G}, \mathrm{~m}^{3} \cdot \mathrm{s}^{-1}$ & $3.4617 \times 10^{-12}$ & $1.3848 \times 10^{-11}$ & $3.1157 \times 10^{-11}$ & $5.5390 \times 10^{-11}$ \\
\hline$\tau=180 \mathrm{~s} \rightarrow Q_{T G}, \mathrm{~m}^{3} \cdot \mathrm{s}^{-1}$ & $1.1533 \times 10^{-12}$ & $4.1650 \times 10^{-12}$ & $1.0387 \times 10^{-11}$ & $1.8463 \times 10^{-11}$ \\
\hline$\tau=600 \mathrm{~s} \rightarrow Q_{T G}, \mathrm{~m}^{3} \cdot \mathrm{s}^{-1}$ & $3.4667 \times 10^{-12}$ & $1.3849 \times 10^{-12}$ & $3.1150 \times 10^{-12}$ & $5.5383 \times 10^{-12}$ \\
\hline
\end{tabular}

Table 5

Volume and volumetric flow rate of the triglyceride reactant fluid for the simulations of the square cross section micro-reactors with $L=233 \times 10^{-4} \mathrm{~m}$.

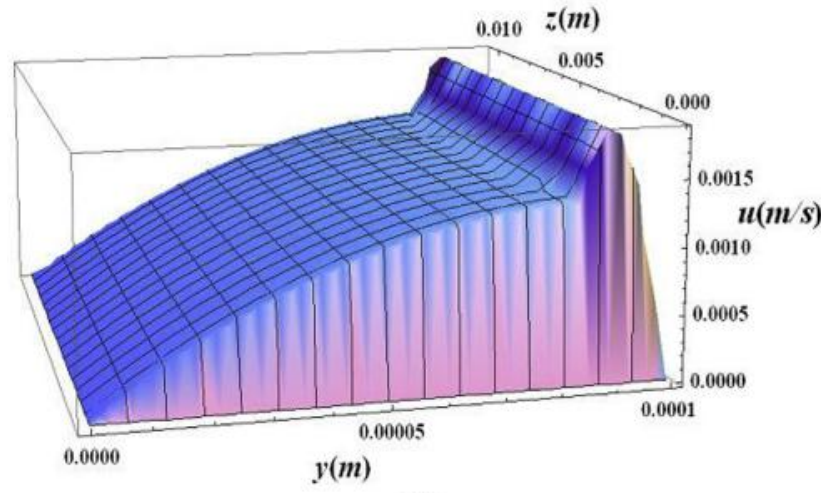

(a)

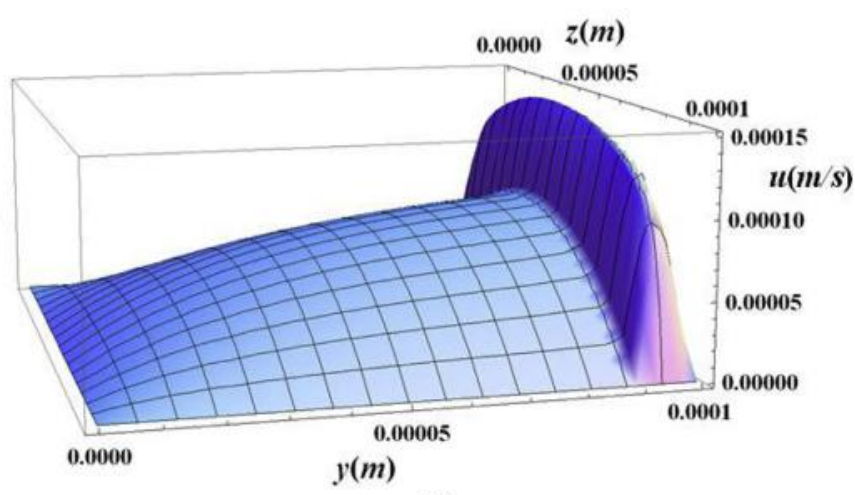

(b)

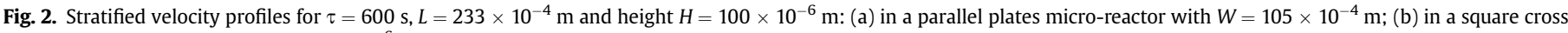
section micro-reactor with $W=100 \times 10^{-6} \mathrm{~m}$. 
Table 6

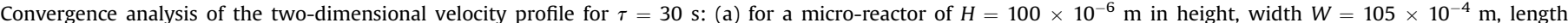

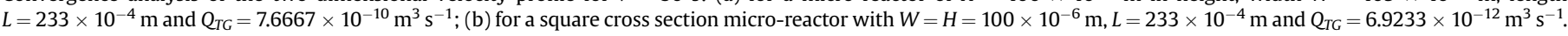

\begin{tabular}{|c|c|c|c|c|c|}
\hline \multicolumn{6}{|c|}{ (a) Parallel Plates Micro-reactor } \\
\hline \multirow[t]{2}{*}{ NT } & \multicolumn{5}{|c|}{$u(y, 0.5 W)$} \\
\hline & $y=10 \times 10^{-6} \mathrm{~m}$ & $y=47 \times 10^{-6} \mathrm{~m}$ & $y=H_{T G}=84.472 \times 10^{-6} \mathrm{~m}$ & $y=90 \times 10^{-6} \mathrm{~m}$ & $y=95 \times 10^{-6} \mathrm{~m}$ \\
\hline 20 & 0.000281 & 0.001033 & 0.001332 & 0.001829 & 0.001354 \\
\hline 40 & 0.000283 & 0.001042 & 0.001343 & 0.001845 & 0.001365 \\
\hline 60 & 0.000284 & 0.001044 & 0.001346 & 0.001849 & 0.001369 \\
\hline 80 & 0.000284 & 0.001045 & 0.001347 & 0.001851 & 0.001370 \\
\hline 100 & 0.000284 & 0.001045 & 0.001347 & 0.001852 & 0.001371 \\
\hline \multicolumn{6}{|c|}{ (b) Square Cross Section Micro-reactor } \\
\hline \multirow{2}{*}{ NT } & \multicolumn{5}{|c|}{$u(y, 0.5 W)$} \\
\hline & $\mathrm{y}=10 \times 10^{-6} \mathrm{~m}$ & $y=50 \times 10^{-6} \mathrm{~m}$ & $y=H_{T G}=89.148 \times 10^{-6} \mathrm{~m}$ & $y=92 \times 10^{-6} \mathrm{~m}$ & $y=98 \times 10^{-6} \mathrm{~m}$ \\
\hline 10 & 0.000442 & 0.001358 & 0.001689 & 0.002861 & 0.001566 \\
\hline 20 & 0.000442 & 0.001358 & 0.001689 & 0.002869 & 0.001573 \\
\hline 30 & 0.000442 & 0.001358 & 0.001689 & 0.002870 & 0.001574 \\
\hline 40 & 0.000442 & 0.001358 & 0.001689 & 0.002870 & 0.001574 \\
\hline
\end{tabular}

this case, it is possible to observe that the velocity profile does not present a uniform distribution in the $\mathrm{z}$ direction, due to significant border effects occurring along this direction. Both figures show that the amplitude of the velocity profile is greater in the region occupied by the alcohol species, due to lower internal resistance that this species presents to flow according with the value of its dynamic viscosity in Table 2. A larger volume of micro-reactor must be occupied by the triglyceride species since this reactant is fed with a volumetric flow rate about 3.402 times greater than the flow rate of the alcohol species.

Table 6 presents the convergence analysis for the velocity profile with different number of terms (NT) in the eigenfunctions expansion at the half the width of the micro-reactor $W(z=0.5 W)$.

Before presenting the results related to triglyceride conversion in micro-reactors, a convergence analysis for the eigenvalues and eigenfunctions obtained from the eigenvalue problem with variable coefficients, for different truncation orders MT of the algebraic system given by Eqs. (C3), must be performed. Tables 7 and 8 present the convergence analysis of the first ten eigenvalues $\beta_{i}$ and $\lambda_{i}$ for the case of $L=233 \times 10^{-4} \mathrm{~m}, H=100 \times 10^{-6} \mathrm{~m}$ and for $W=105 \times 10^{-4} \mathrm{~m}$ and $W=100 \times 10^{-6} \mathrm{~m}$, respectively. The first eigenvalue $\lambda_{1}=0$ has been omitted in such tables, since it is exactly recovered in all cases. It is possible to check in Table 7 that the first ten and most important eigenvalues present at least three converged significant digits for a truncation order as low as MT $=60$ or greater, while that in Table 8 these eigenvalues present three converged significant digits for a truncation order MT $=100$.

Figs. 3 and 4 illustrate the convergence behavior of the tenth eigenfunction as a solution of the eigenvalue problems obtained by GITT, for the eigenfunctions $\Gamma_{i}(Y, Z)$ and $\Psi_{i}(Y, Z)$, with different truncation orders MT for the case of $L=233 \times 10^{-4} \mathrm{~m}$, $H=100 \times 10^{-6} \mathrm{~m}$ and for $W=105 \times 10^{-4} \mathrm{~m}$ and $W=100 \times 10^{-6} \mathrm{~m}$,

Table 7

Convergence analysis of the first ten eigenvalues $\beta_{i}$ and $\lambda_{i}$ for $L=233 \times 10^{-4} \mathrm{~m}, H=100 \times 10^{-6} \mathrm{~m}$ and $W=105 \times 10^{-4} \mathrm{~m}$.

\begin{tabular}{lccccc}
\hline$\beta_{\boldsymbol{i}}$ & $\mathbf{M T}=\mathbf{2 0}$ & $\mathbf{M T}=\mathbf{4 0}$ & $\mathbf{M T}=\mathbf{6 0}$ & $\mathbf{M T}=\mathbf{8 0}$ & $\mathbf{M T}=\mathbf{1 0 0}$ \\
\hline 1 & 1.858231 & 1.858223 & 1.858221 & 1.858221 & 1.858220 \\
2 & 3.912254 & 3.912179 & 3.912149 & 3.912130 & 3.912130 \\
3 & 5.152310 & 5.152067 & 5.152025 & 5.152010 & 5.151993 \\
4 & 6.340924 & 6.340519 & 6.340385 & 6.340300 & 6.340296 \\
5 & 6.520034 & 6.519768 & 6.519646 & 6.519645 & 6.519571 \\
6 & 8.427524 & 8.425907 & 8.425676 & 8.425558 & 8.425495 \\
7 & 8.697742 & 8.694679 & 8.694380 & 8.694343 & 8.694179 \\
8 & 9.057202 & 9.055777 & 9.055245 & 9.054957 & 9.054957 \\
9 & 9.236979 & 9.234651 & 9.234191 & 9.233922 & 9.233904 \\
10 & 11.140860 & 11.126605 & 11.125711 & $\mathbf{M T}=\mathbf{8 0}$ & $\mathbf{1 1 2 5 2 4 0}$ \\
\hline$\lambda_{\boldsymbol{i}}$ & $\mathbf{M T}=\mathbf{2 0}$ & $\mathbf{M T}=\mathbf{4 0}$ & $\mathbf{M T}=\mathbf{6 0}$ & 2.952109 & $\mathbf{M T}=\mathbf{1 0 0}$ \\
\hline 2 & 2.952168 & 2.952133 & 2.952118 & 2.952109 \\
3 & 3.503921 & 3.503817 & 3.503805 & 5.038533 & 3.503791 \\
4 & 5.038861 & 5.038643 & 5.038569 & 5.549706 & 5.038531 \\
5 & 5.549969 & 5.549792 & 5.549744 & 6.787071 & 5.549654 \\
6 & 6.788439 & 6.787300 & 6.787139 & 7.559532 & 6.787032 \\
7 & 7.561511 & 7.559735 & 7.559690 & 7.748215 & 7.559424 \\
8 & 7.750105 & 7.748620 & 7.748285 & 8.102660 & 7.748132 \\
9 & 8.104429 & 8.103304 & 9.102886 & 10.050262 & 8.102660 \\
10 & 9.993524 & 9.886695 & 10.050569 & 9.885704 \\
11 & 10.060303 & 10.051266 & & 10.050197 \\
\hline
\end{tabular}


Table 8

Convergence analysis of the first ten eigenvalues $\beta_{i}$ and $\lambda_{i}$ for $L=233 \times 10^{-4} \mathrm{~m}, W=H=100 \times 10^{-6} \mathrm{~m}$.

\begin{tabular}{lccccc}
\hline$\beta_{\boldsymbol{i}}$ & $\mathbf{M T}=\mathbf{2 0}$ & $\mathbf{M T}=\mathbf{4 0}$ & $\mathbf{M T}=\mathbf{6 0}$ & $\mathbf{M T}=\mathbf{8 0}$ & $\mathbf{M T}=\mathbf{1 0 0}$ \\
\hline 1 & 1.792537 & 1.792502 & 1.792497 & 1.792496 & 1.792495 \\
2 & 4.458316 & 4.456611 & 4.456364 & 4.456305 & 4.456303 \\
3 & 4.848068 & 4.847499 & 4.847447 & 4.847421 & 4.847410 \\
4 & 6.897659 & 6.895489 & 6.895066 & 6.894934 & 6.894921 \\
5 & 7.328126 & 7.324468 & 7.323912 & 7.323904 & 7.323755 \\
6 & 7.659307 & 7.646321 & 7.646040 & 7.645942 & 7.645901 \\
7 & 9.573535 & 9.520232 & 9.519339 & 9.519299 & 9.519112 \\
8 & 9.954042 & 9.549954 & 9.549348 & 9.549160 & 9.549124 \\
9 & 10.987692 & 10.117503 & 10.117001 & 10.116148 & 10.116145 \\
10 & 11.068464 & 10.362397 & 10.361016 & 10.360666 & 10.360579 \\
\hline$\lambda_{\boldsymbol{i}}$ & $\mathbf{M T}=\mathbf{2 0}$ & $\mathbf{M T}=\mathbf{4 0}$ & $\mathbf{M T}=\mathbf{6 0}$ & $\mathbf{M T}=\mathbf{8 0}$ & $\mathbf{M T}=\mathbf{1 0 0}$ \\
\hline 2 & 3.346871 & 3.346480 & 3.346458 & 3.346441 & 3.346435 \\
3 & 3.489820 & 3.488618 & 3.488456 & 5.577561 & 3.488420 \\
4 & 5.580246 & 5.578045 & 5.577650 & 6.233550 & 5.577553 \\
5 & 6.235656 & 6.233840 & 6.233598 & 6.329554 & 6.233523 \\
6 & 6.336109 & 6.330057 & 6.329758 & 8.213979 & 8.329426 \\
7 & 8.222284 & 8.214905 & 8.214090 & 8.355723 & 8.9973919 \\
8 & 8.645461 & 8.356421 & 8.356176 & 9.066560 & 10.698737 \\
9 & 9.467910 & 8.997967 & 9.997577 & & 9.9973543 \\
10 & 9.742186 & 9.068641 & 10.699268 & & 10.698539 \\
11 & 11.577117 & 10.702218 & & &
\end{tabular}
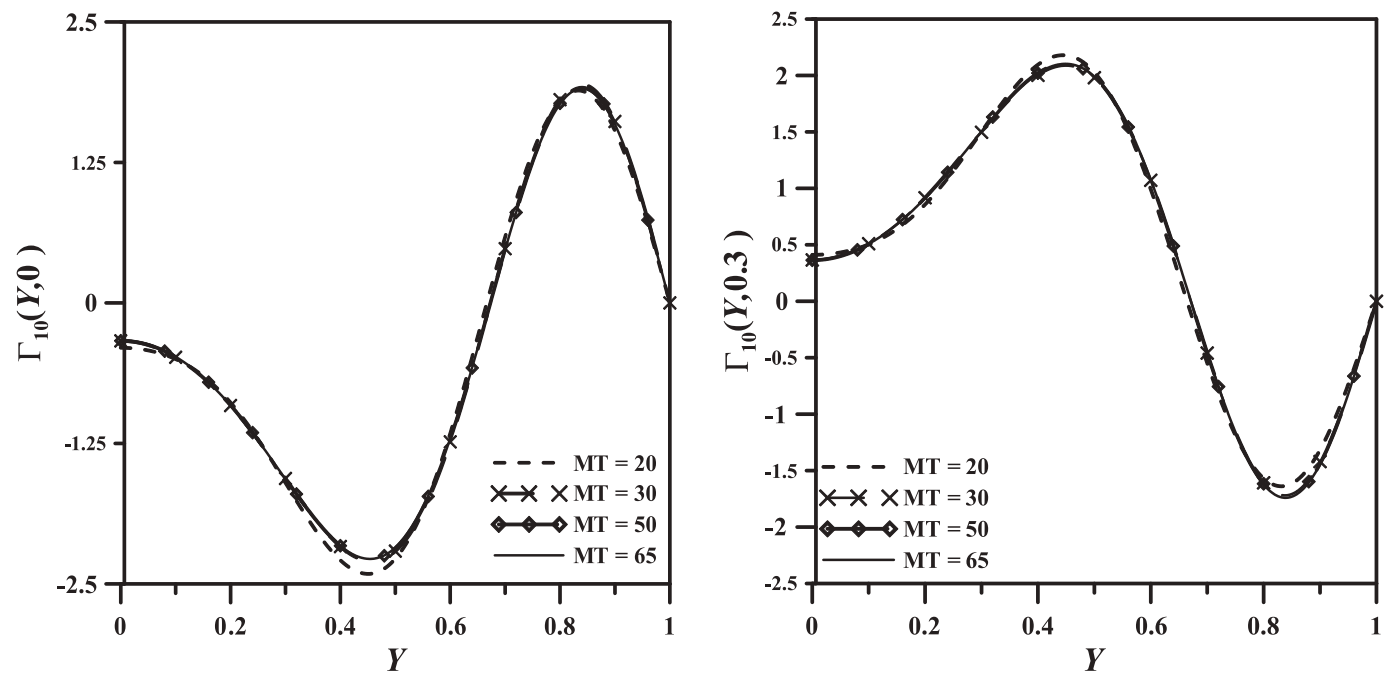

(a)
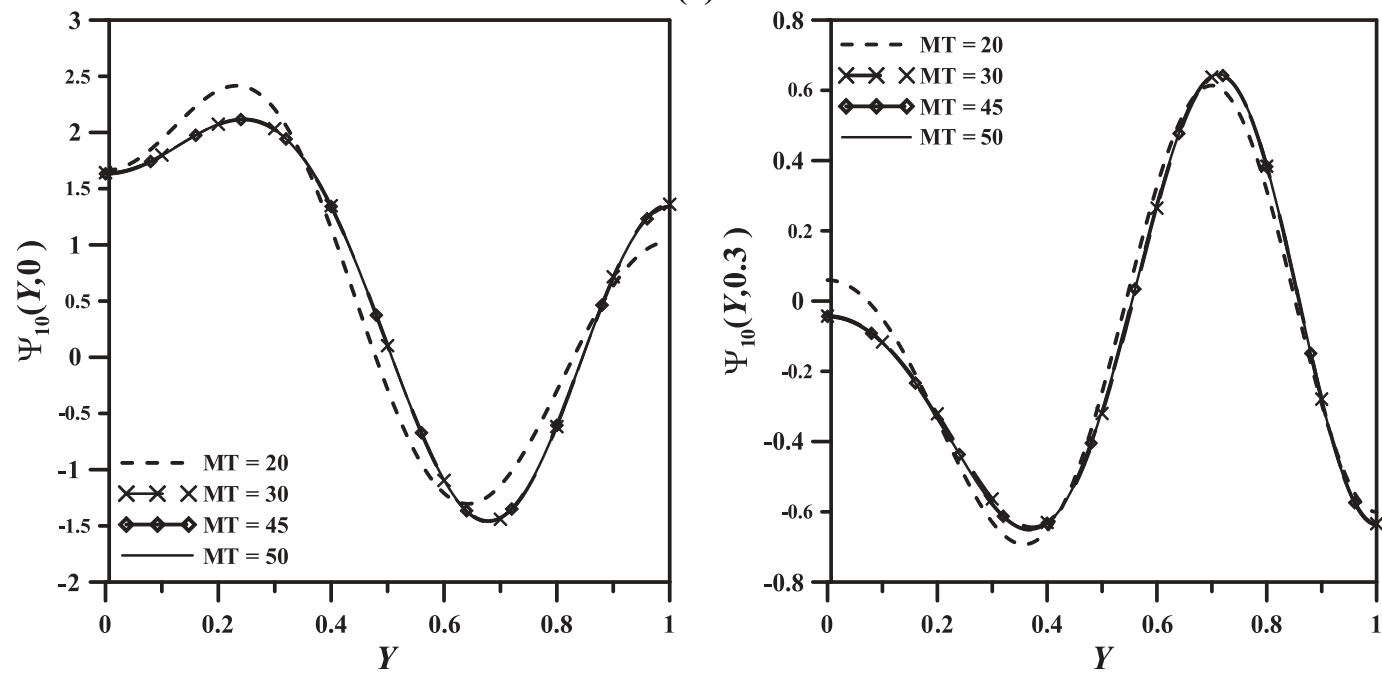

(b)

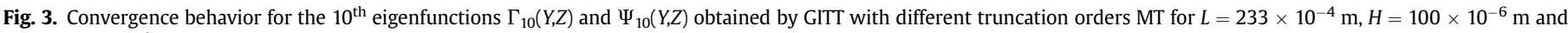
$W=105 \times 10^{-4} \mathrm{~m}$. 

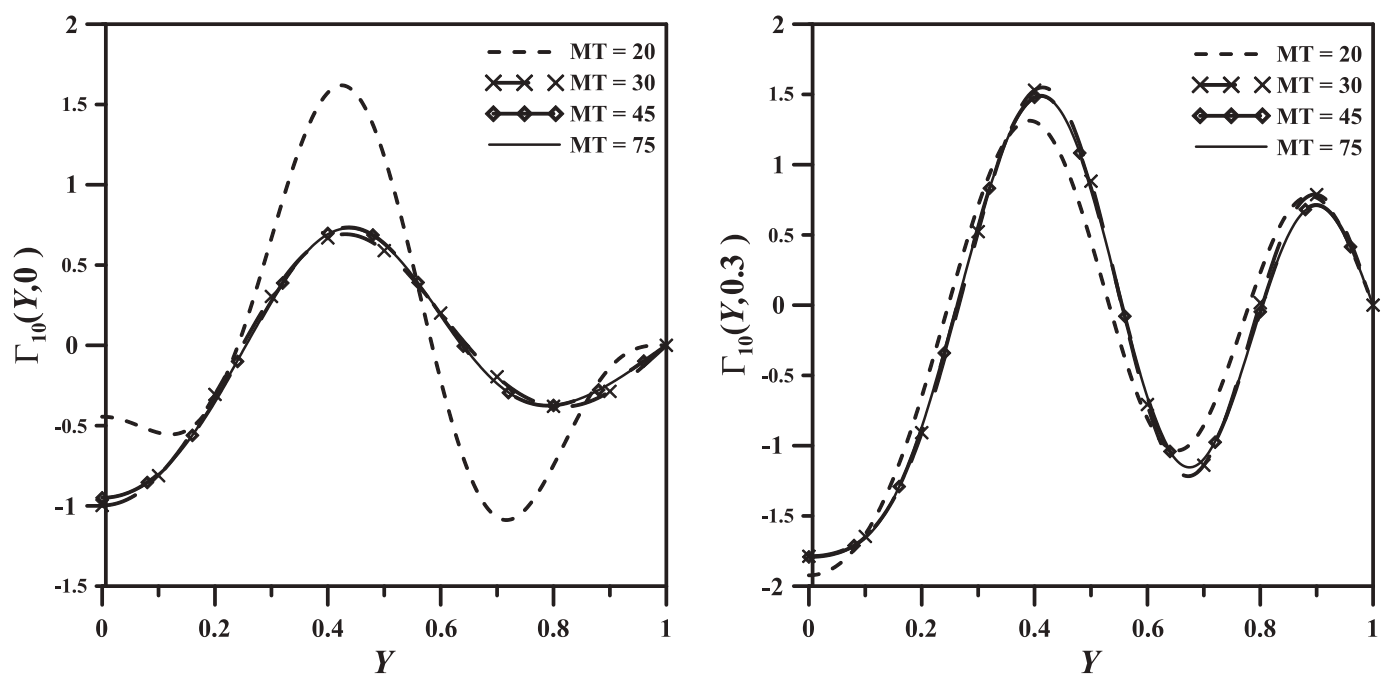

(a)
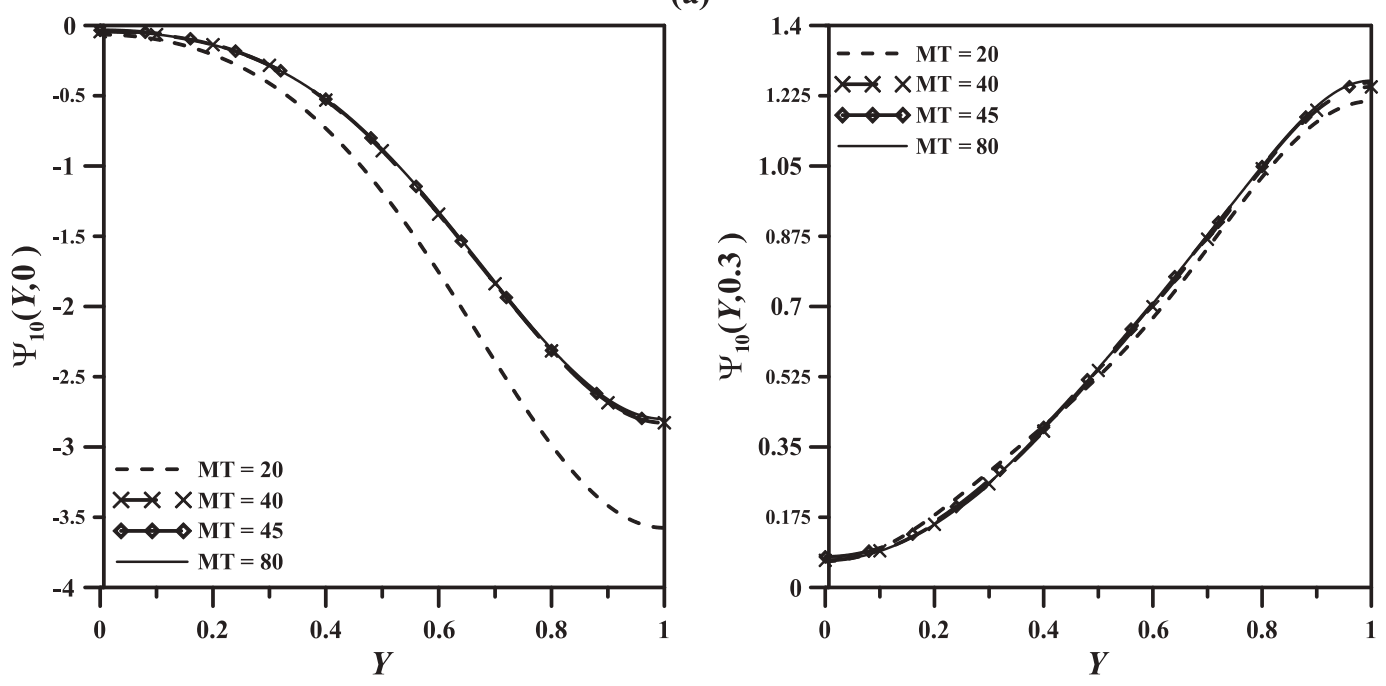

(b)

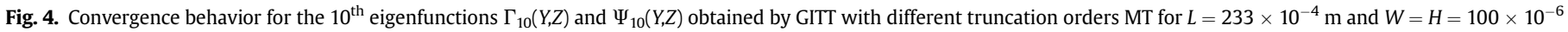
$\mathrm{m}$.

respectively. It is possible to observe that for $\mathrm{MT}=30$ both eigenfunctions are fully converged on the graph scale for $W=105 \times 10^{-4} \mathrm{~m}$, while for $W=100 \times 10^{-6} \mathrm{~m}$ the eigenfunctions are converged with $\mathrm{MT}=45$. It is important to observe that for different values of $W$, both eigenvalues and eigenfunctions change, evidencing the sensitivity of the eigenvalue problem to changes in the velocity profile.

Table 9 presents the convergence analysis of the average concentrations for the triglyceride and biodiesel species in microreactors at $T=298 \mathrm{~K}$ and $\tau=30 \mathrm{~s}$. In both cases, it can be seen that the solution obtained by GITT through the eigenvalue problems with variable coefficients, converges with up to four significant digits with only 50 terms, whereas for the eigenvalue problems with constant coefficients 100 terms or more are needed. These results were expected, since the velocity profile information was introduced into eigenvalues and eigenfunctions obtained from the eigenvalue problems with variable coefficients, while the eigenvalue problems with constant coefficients contain only the information of diffusive effects.

Fig. 5 shows the dimensionless concentration profiles of triglyceride and biodiesel along a micro-reactor with $L=233 \times 10^{-4} \mathrm{~m}, W=105 \times 10^{-4} \mathrm{~m}$ and $H=100 \times 10^{-6} \mathrm{~m}$ for different residence times at $298 \mathrm{~K}$. It is observed a good agreement between the results obtained by GITT for the eigenvalue problems with both variable coefficients (variable EP) and constant coefficients (constant EP), against those from the literature [13,39]. Larger triglyceride consumption in the transesterification reaction occurs for longer residence times or increased exposure times of reagent fluids to reactive, diffusive and convective effects, resulting in a more noticeable reduction in the average concentration of triglyceride and, due to the reaction stoichiometry, in the increased formation of biodiesel along the micro-reactor to reach the equilibrium condition.

Fig. 6a,b illustrates, respectively, the temperature effects in a micro-reactor with fixed dimensions $L=233 \times 10^{-4} \mathrm{~m}$, $W=105 \times 10^{-4} \mathrm{~m}$ and $H=100 \times 10^{-6} \mathrm{~m}$, and the effect of the micro-reactor height at $298 \mathrm{~K}$, on the triglyceride conversion. Fig. 6a presents the influence of temperature on the triglyceride conversion rate up to $333 \mathrm{~K}$, near the boiling point of methanol. The temperature influences the kinetics constants of the 
Table 9

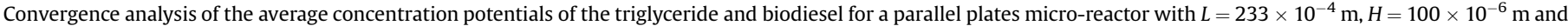
$W=105 \times 10^{-4} \mathrm{~m}$, and for a square cross section micro-reactor with $L=233 \times 10^{-4} \mathrm{~m}, W=H=100 \times 10^{-6} \mathrm{~m}$.

\begin{tabular}{|c|c|c|c|c|c|c|}
\hline \multicolumn{7}{|c|}{ Parallel Plates Micro-reactor } \\
\hline \multicolumn{7}{|c|}{ Eigenvalue problem with constant coefficients } \\
\hline Point & \multicolumn{2}{|c|}{$X=0.3$} & \multicolumn{2}{|c|}{$X=0.5$} & \multicolumn{2}{|c|}{$X=0.7$} \\
\hline NT & $C_{T G, A v}$ & $C_{B, A v}$ & $C_{T G, A v}$ & $C_{B, A v}$ & $C_{T G, A v}$ & $C_{B, A v}$ \\
\hline 20 & 0.961441 & 0.041956 & 0.914454 & 0.102134 & 0.859002 & 0.186241 \\
\hline 40 & 0.961619 & 0.041748 & 0.914660 & 0.101849 & 0.859218 & 0.185888 \\
\hline 60 & 0.961736 & 0.041613 & 0.914804 & 0.101649 & 0.859375 & 0.185632 \\
\hline 80 & 0.961729 & 0.041620 & 0.914792 & 0.101666 & 0.859359 & 0.185657 \\
\hline 100 & 0.961695 & 0.041659 & 0.914748 & 0.101727 & 0.859310 & 0.185737 \\
\hline \multicolumn{7}{|c|}{ Eigenvalue problem with variable coefficients } \\
\hline Point & \multicolumn{2}{|c|}{$X=0.3$} & \multicolumn{2}{|c|}{$X=0.5$} & \multicolumn{2}{|c|}{$X=0.7$} \\
\hline NT & $C_{T G, A v}$ & $C_{B, A v}$ & $C_{T G, A v}$ & $C_{B, A v}$ & $C_{T G, A v}$ & $C_{B, A v}$ \\
\hline 10 & 0.961733 & 0.041618 & 0.914777 & 0.101685 & 0.859324 & 0.185707 \\
\hline 20 & 0.961715 & 0.041636 & 0.914768 & 0.101697 & 0.859327 & 0.185707 \\
\hline 30 & 0.961676 & 0.041682 & 0.914732 & 0.101748 & 0.859294 & 0.185760 \\
\hline 40 & 0.961717 & 0.041634 & 0.914771 & 0.101693 & 0.859333 & 0.185699 \\
\hline 50 & 0.961703 & 0.041651 & 0.914759 & 0.101711 & 0.859322 & 0.185717 \\
\hline \multicolumn{7}{|c|}{ Square Cross Section Micro-reactor } \\
\hline \multicolumn{7}{|c|}{ Eigenvalue problem with constant coefficients } \\
\hline Point & \multicolumn{2}{|c|}{$X=0.3$} & \multicolumn{2}{|c|}{$X=0.5$} & \multicolumn{2}{|c|}{$X=0.7$} \\
\hline NT & $C_{T G, A v}$ & $C_{B, A v}$ & $C_{T G, A v}$ & $C_{B, A v}$ & $C_{T G, A v}$ & $C_{B, A v}$ \\
\hline 20 & 0.955411 & 0.049206 & 0.904022 & 0.117023 & 0.844553 & 0.210476 \\
\hline 40 & 0.955648 & 0.048923 & 0.904303 & 0.116622 & 0.844850 & 0.209973 \\
\hline 60 & 0.955815 & 0.048725 & 0.904513 & 0.116322 & 0.845079 & 0.209585 \\
\hline 80 & 0.955802 & 0.048740 & 0.904492 & 0.116351 & 0.845054 & 0.209627 \\
\hline 100 & 0.955752 & 0.048799 & 0.904426 & 0.116445 & 0.844981 & 0.209751 \\
\hline \multicolumn{7}{|c|}{ Eigenvalue problem with variable coefficients } \\
\hline Point & \multicolumn{2}{|c|}{$X=0.3$} & \multicolumn{2}{|c|}{$X=0.5$} & \multicolumn{2}{|c|}{$X=0.7$} \\
\hline NT & $C_{T G, A v}$ & $C_{B, A v}$ & $C_{T G, A v}$ & $C_{B, A v}$ & $C_{T G, A v}$ & $C_{B, A v}$ \\
\hline 10 & 0.955449 & 0.049150 & 0.904122 & 0.116853 & 0.844666 & 0.210240 \\
\hline 20 & 0.955705 & 0.048851 & 0.904378 & 0.116504 & 0.844923 & 0.209831 \\
\hline 30 & 0.955751 & 0.048798 & 0.904427 & 0.116438 & 0.844977 & 0.209748 \\
\hline 40 & 0.955761 & 0.048785 & 0.904438 & 0.116423 & 0.844988 & 0.209729 \\
\hline 50 & 0.955766 & 0.048781 & 0.904444 & 0.116415 & 0.844997 & 0.209717 \\
\hline
\end{tabular}

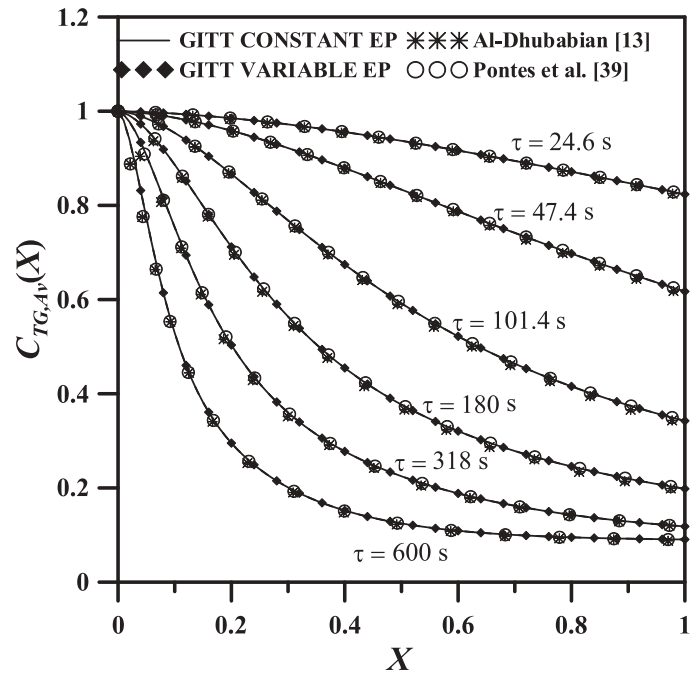

(a)

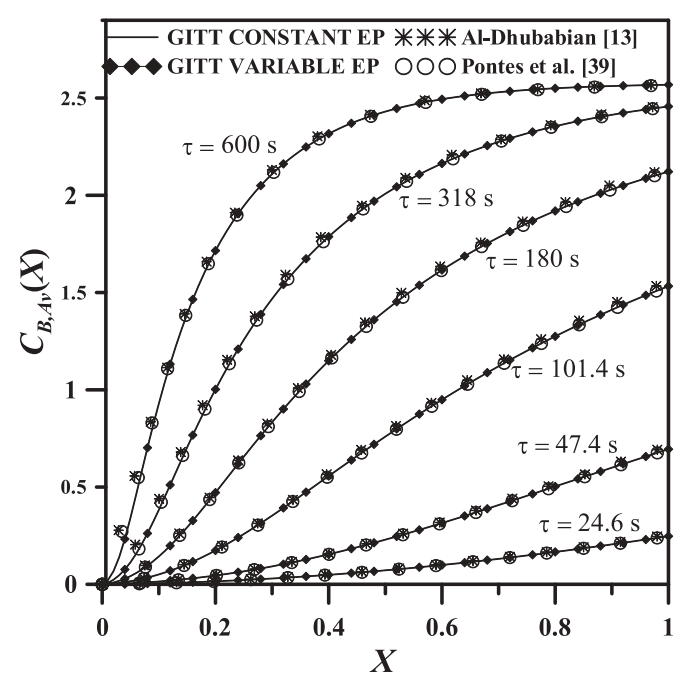

(b)

Fig. 5. Dimensionless average concentrations along the micro-reactor with $L=233 \times 10^{-4} \mathrm{~m}, H=100 \times 10^{-6} \mathrm{~m}$ and $W=105 \times 10^{-4} \mathrm{~m}$ : (a) triglyceride; (b) biodiesel. 


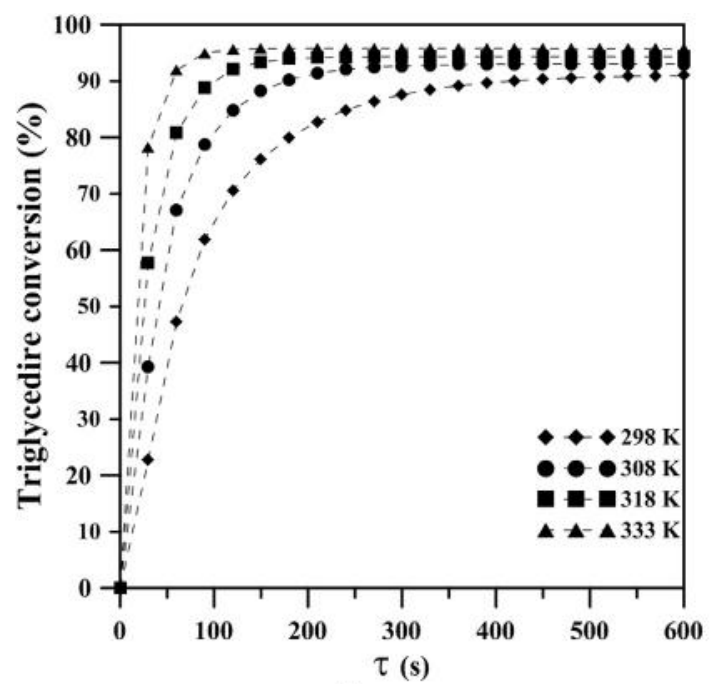

(a)

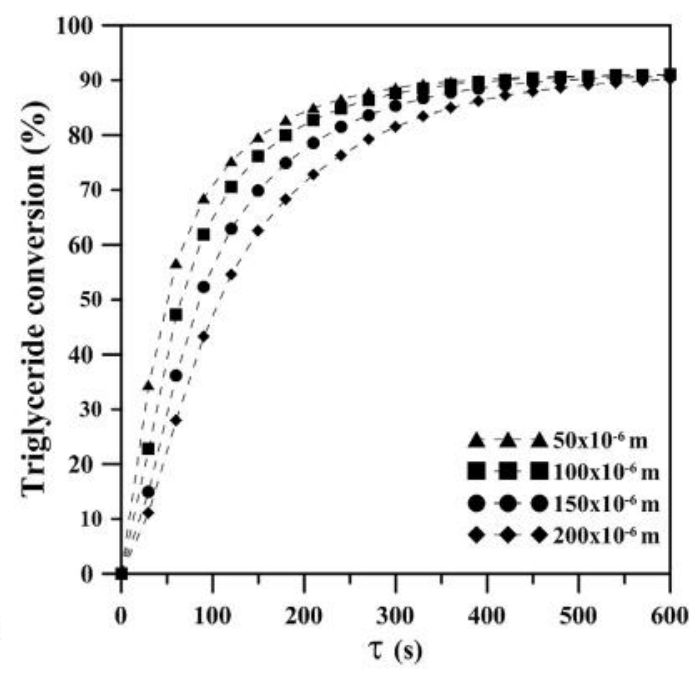

(b)

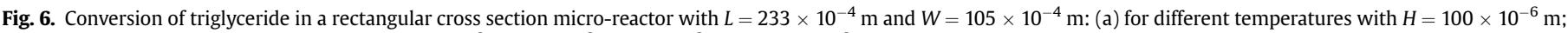
(b) different micro-reactor heights, i.e., $H=50 \times 10^{-6}, 100 \times 10^{-6}, 150 \times 10^{-6}$ and $200 \times 10^{-6} \mathrm{~m}$ at $298 \mathrm{~K}$.

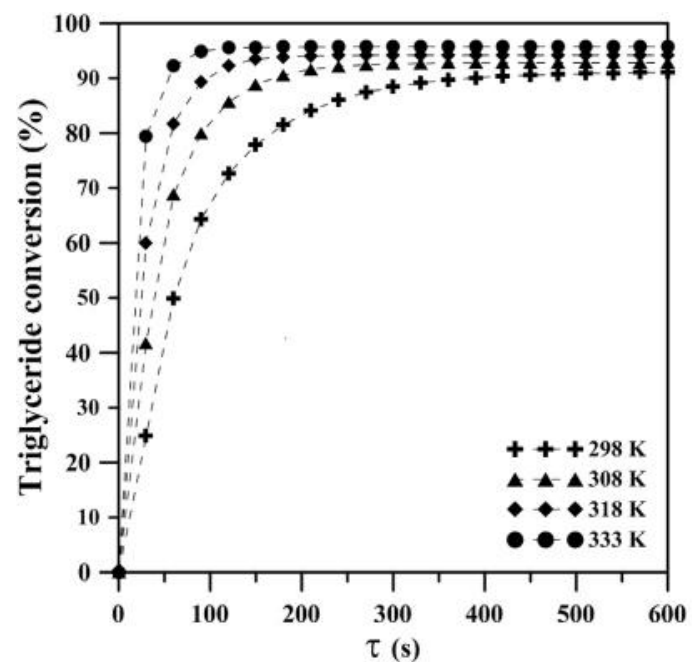

(a)

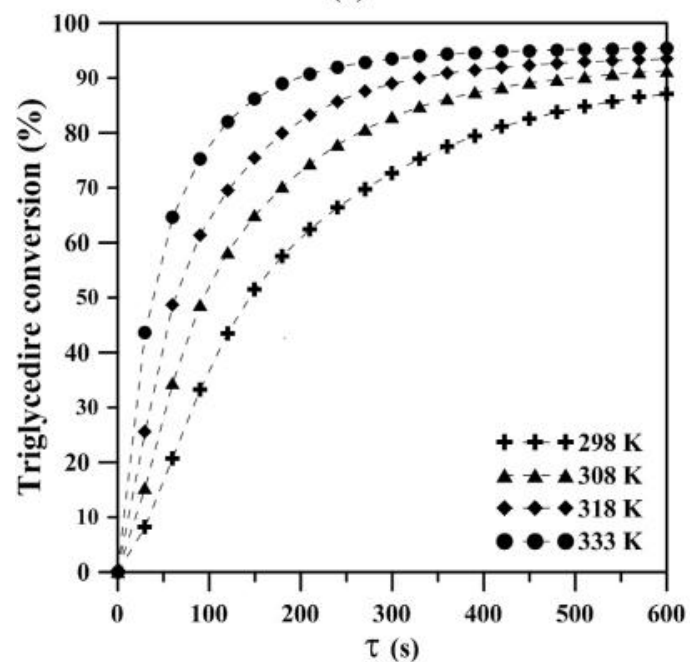

(c)

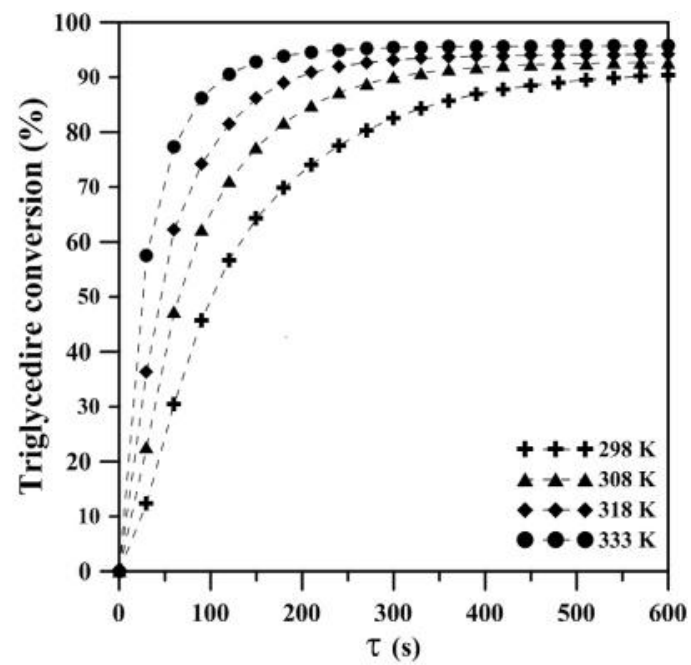

(b)

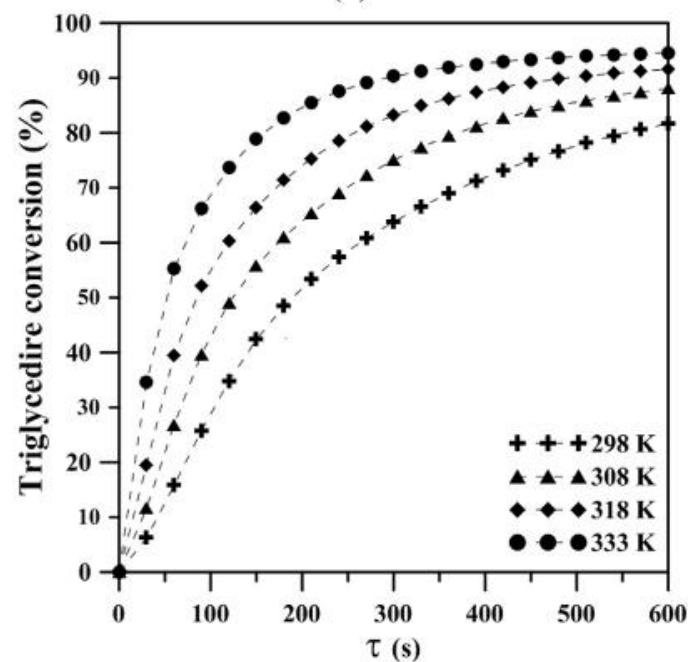

(d)

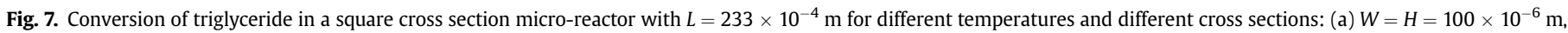
(b) $W=H=200 \times 10^{-6} \mathrm{~m}$, (c) $W=H=300 \times 10^{-6} \mathrm{~m}$ and (d) $W=H=400 \times 10^{-6} \mathrm{~m}$. 
transesterification process, which as a consequence, influence the reaction process, achieving higher conversion rates in shorter residence times. For a given microchannel height, four temperatures were studied, and higher temperatures yielded higher and faster conversions. Fig. $6 \mathrm{~b}$ presents a comparison between the conversion of triglyceride for four different micro-reactor heights, i.e., $50 \times 10^{-6}, 100 \times 10^{-6}, 150 \times 10^{-6}$ and $200 \times 10^{-6} \mathrm{~m}$. The microreactor of $50 \times 10^{-6} \mathrm{~m}$ in height yielded a higher conversion than all the other micro-reactors for any given residence time. This occurs because lower micro-reactor heights result in higher surface area to volume ratio and shorter diffusion pathways, ensuring greater mass transfer and better triglyceride conversions. The same results are observed for the square cross section micro-reactors illustrated in Fig. 7 for different cross sections and different temperatures. Comparing the graphics, it is noticeable that the best results for the conversion of triglyceride for a given residence time are expected at the lower square cross section micro-reactors, $H=W=100 \times 10^{-6} \mathrm{~m}$, and at greater reaction temperature, $T=333 \mathrm{~K}$.

\section{Conclusions}

A theoretical analysis of the biodiesel synthesis from methanol and soybean oil, catalyzed by sodium hydroxide in rectangular cross section micro-reactors, is here presented. A threedimensional reaction-convection-diffusion model for the mass transfer of the species involved in the transesterification is adopted, considering a second order reversible and homogeneous chemical reaction and fully developed stratified laminar two-phase flow. A hybrid numerical-analytical solution is then proposed based on the Generalized Integral Transform Technique (GITT).

Two eigenvalue problems were considered in the solution methodology, one accounting for the velocity profile and another with constant coefficients. The results indicate better convergence behavior for the solutions obtained through the eigenvalue problems with variable coefficients, which were solved by the GITT itself. Results for the hybrid numerical-analytical concentration fields were confronted with data from the literature for typical situations with good adherence among them.

Important parameters such as the reaction temperature, residence time and volumetric flow rate of the triglyceride species, and dimensions of the micro-reactor are analyzed, aiming at studying the influence of each parameter in the transesterification reaction. The results obtained confirm the expected behavior that enhanced conversions of triglyceride occur for higher residence time, larger reaction temperature and lower height of the micro-reactor. In these reactive conditions the reactant fluids would be for a longer time in contact, favoring the chemical interactions via a greater surface area to volume ratios, and with the reaction rates increased by the temperature effects.

\section{Acknowledgements}

The authors would like to acknowledge the financial support provided by FAPERJ, CNPq and CAPES, all of them research governmental sponsoring agencies in Brazil.

\section{Appendix A. Solution methodology for the velocity field}

The appropriate eigenvalue problem to represent the velocity eigenfunction expansion in the $z$ direction can be written as: $\frac{d^{2} \Psi_{v e l, i}(z)}{d z^{2}}+\lambda_{i}^{2} \Psi_{v e l, i}(z)=0$

$\Psi_{\nu e l, i}(0)=0$

$\Psi_{v e l, i}(W)=0$

The eigenvalue problem defined above has a known solution in the literature [50] and the normalized eigenfunction $\tilde{\Psi}$ and its norm and eigenvalues can be written as:

$\tilde{\Psi}_{v e l, i}(z)=\frac{\sin \left(\lambda_{i} z\right)}{\sqrt{N_{v e l, i}}}$
$N_{v e l, i}=\frac{W}{2}$
$\lambda_{i}=\frac{i \pi}{W}, i=1,2,3, \ldots$

From the orthogonality property of the eigenfunctions, the following transform and inverse formulae for the velocity fields of the two fluids are presented in Eqs. (A2.a,b) and (A2.c,d), respectively:

$$
\begin{aligned}
& \bar{u}_{T G, i}(y)=\int_{0}^{W} u_{T G}(y, z) \tilde{\Psi}_{v e l, i}(z) d z \\
& \bar{u}_{A, i}(y)=\int_{0}^{W} u_{A}(y, z) \tilde{\Psi}_{v e l, i}(z) d z \\
& u_{T G}(y, z)=\sum_{i=1}^{\infty} \tilde{\Psi}_{v e l, i}(z) \bar{u}_{T G, i}(y) \\
& u_{A}(y, z)=\sum_{i=1}^{\infty} \tilde{\Psi}_{v e l, i}(z) \bar{u}_{A, i}(y)
\end{aligned}
$$

The integral transformation of Eqs. (2. a,b) can be carried out by application of the operator $\int_{0}^{W} \tilde{\Psi}_{v e l, i}(z)_{-\_} d z$ together with the boundary conditions of the original velocity problem, resulting in the following transformed system:

$$
\frac{d^{2} \bar{u}_{T G, i}}{d y^{2}}-\lambda_{i}^{2} \bar{u}_{T G, i}(y)=-\frac{1}{\mu_{T G}} \frac{\Delta P}{L} \int_{0}^{W} \tilde{\Psi}_{v e l, i}(z) d z, \quad 0<y<H_{T G}
$$

$\frac{d^{2} \bar{u}_{A, i}}{d y^{2}}-\lambda_{i}^{2} \bar{u}_{A, i}(y)=-\frac{1}{\mu_{A}} \frac{\Delta P}{L} \int_{0}^{W} \tilde{\Psi}_{v e l, i}(z) d z, \quad H_{T G}<y<H$

$\bar{u}_{T G, i}(0)=0 ;$
$\bar{u}_{A, i}(H)=0$

$\bar{u}_{T G, i}\left(H_{T G}\right)=\bar{u}_{A, i}\left(H_{T G}\right)$

$-\left.\mu_{T G} \frac{d \bar{u}_{T G, i}}{d y}\right|_{y=H_{T G}}=-\left.\mu_{A} \frac{d \bar{u}_{A, i}}{d y}\right|_{y=H_{T G}}$

Equations (A3) form an infinite system of second order ordinary differential equations coupled at the interface conditions at $y=H_{T G}$. 
Such equations can be solved analytically [51] and their solutions are then used to explicitly represent the velocity fields of the two fluids through the inversion formulae given by Eqs. (A2.c,d). Therefore, the velocity profile of the two fluids, methanol and soybean oil, can be written in terms of hyperbolic functions according to Eqs. (4a-c).

\section{Appendix B. Solution methodology for the concentration field with simple eigenvalue problem}

First, the auxiliary problems with constant coefficients are considered, without the velocity profile. Thus, two eigenvalue problems must be proposed in order to automatically satisfy the different boundary conditions of the partial differential equations in the $Y$ and $Z$ directions, one for the alcohol and another one for the remaining species. Table B1 presents the proposed eigenvalue problems:

Both eigenvalue problems can be solved by the separation of variables technique [50]. For this purpose, two new auxiliary problems are required to represent the original eigenfunction $\Phi(Y, Z)$ and $\Omega(Y, Z)$, which are presented in Table B2, respectively.

From the auxiliary eigenvalue problems proposed in Table B2, it is possible to express the normalized eigenfunctions $\tilde{\Phi}$ and $\tilde{\Omega}$ and their respective eigenvalues as:

$$
\begin{aligned}
& \tilde{\Phi}_{m, n}(Y, Z)=\tilde{\phi}_{1, m}(Y) \tilde{\phi}_{2, n}(Z) ; \\
& \widetilde{\Omega}_{m, n}(Y, Z)=\tilde{\varphi}_{1, m}(Y) \tilde{\varphi}_{2, n}(Z) \\
& \gamma_{m, n}^{2}=\gamma_{1, m}^{2}+\gamma_{2, n}^{2} ; \\
& v_{m, n}^{2}=v_{1, m}^{2}+v_{2, n}^{2}
\end{aligned}
$$

A reordering scheme for the eigenvalues and eigenfunctions can be proposed in order to ensure computational efficiency [35]. Thus, the normalized eigenfunctions $\tilde{\Phi}_{m, n}(Y, Z)$ and $\tilde{\Omega}_{m, n}(Y, Z)$ are then converted to eigenfunctions with simple index $\tilde{\Phi}_{i}(Y, Z)$ and $\tilde{\Omega}_{i}(Y, Z)$, where the index $i$ corresponds to combinations of the indices of the auxiliary eigenvalue problems $m$ and $n$ rearranged in ascending order of the sum of the squares of these eigenvalues in each direction. The objective of this strategy is to account only for the most important terms, in decreasing order of importance in the sum represented by the eigenfunction expansion, reducing the double summation to a single one, and thus significantly reducing the computational cost in the solution of the transformed system.

After the solution of the eigenvalue problem, the transform and inverse formulae for the concentration of all species involved in the transesterification reaction can be defined in Eqs. (B2,B3), respectively, as:

$$
\begin{aligned}
& \bar{C}_{A h_{i}}(X)=\int_{0}^{1} \int_{0}^{1} \tilde{\Phi}_{i}(Y, Z) C_{A h}(X, Y, Z) d Y d Z ; \\
& \bar{C}_{r_{i}}(X)=\int_{0}^{1} \int_{0}^{1} \tilde{\Omega}_{i}(Y, Z) C_{r}(X, Y, Z) d Y d Z \\
& C_{A h}(X, Y, Z)=\sum_{i=1}^{\infty} \tilde{\Phi}_{i}(Y, Z) \bar{C}_{A h_{i}}(X) ; \\
& C_{r}(X, Y, Z)=\sum_{i=1}^{\infty} \tilde{\Omega}_{i}(Y, Z) \bar{C}_{r_{i}}(X)
\end{aligned}
$$

with $r=T G, D G, M G, B, G L$.

The integral transformation of the filtered partial differential equations, together with their boundary conditions in the direction $X$, can be carried out operating with the integral operator of the respective eigenfunctions for each species over the $Y$ and $Z$ domain, together with the second Green's formula, resulting in the transformed system defined by Eqs. (B4):

$$
\sum_{j=1}^{\infty} O_{1, i j} \frac{d \bar{C}_{r_{j}}}{d X}=\frac{1}{P e_{r}} \frac{d^{2} \bar{C}_{r_{i}}}{d X^{2}}-\xi_{r} v_{i}^{2} \bar{C}_{r_{i}}+\varsigma \bar{G}_{r, i j k},
$$

where $r=T G, D G, M G, B, G L$

$\sum_{j=1}^{\infty} O_{2, i j} \frac{d \bar{C}_{A h_{j}}}{d X}=\frac{1}{P e_{A h}} \frac{d^{2} \bar{C}_{A h_{i}}}{d X^{2}}-\xi_{A h} \gamma_{i}^{2} \bar{C}_{A h_{i}}+\varsigma \bar{G}_{A h, i j k}$

$\bar{C}_{T G_{i}}(0)=\bar{f}_{1, i}$

$\bar{C}_{A h_{i}}(0)=\bar{f}_{2, i}$;

$\bar{C}_{r_{i}}(0)=0$,

where $r=D G, M G, B, G L$

$\left.\frac{d \bar{C}_{r_{i}}}{d X}\right|_{X=1}=0$, where $r=T G, A, D G, M G, B, G L$

where the transformed kinetic expressions are presented in Table B3.

The various coefficients can be analytically determined via symbolic computation [51] and are defined as:

$$
\begin{aligned}
& O_{1, i j}=\int_{0}^{1} \int_{0}^{1} U_{T G} \widetilde{\Omega}_{i} \widetilde{\Omega}_{j} d Y d Z ; \quad O_{2, i j}=\int_{0}^{1} \int_{0}^{1} U_{T G} \widetilde{\Phi}_{i} \widetilde{\Phi}_{j} d Y d Z ; \quad O_{3, i j k}=\int_{0}^{1} \int_{0}^{1} \widetilde{\Omega}_{i} \widetilde{\Omega}_{j} \widetilde{\Phi}_{k} d Y d Z ; \\
& O_{4, j k}=\int_{0}^{1} \int_{0}^{1} \widetilde{\Omega}_{i} \widetilde{\Omega}_{j} \widetilde{\Omega}_{k} d Y d Z ; \quad O_{5, i j k}=\int_{0}^{1} \int_{0}^{1} \widetilde{\Phi}_{i} \widetilde{\Omega}_{j} \widetilde{\Phi}_{k} d Y d Z ; \quad O_{6, i j k}=\int_{0}^{1} \int_{0}^{1} \widetilde{\Phi}_{i} \widetilde{\Omega}_{j} \widetilde{\Omega}_{k} d Y d Z ; \\
& O_{7, i j}=\int_{0}^{1} \int_{0}^{1} \widetilde{\Phi}_{i} \widetilde{\Omega}_{j} d Y d Z ; \bar{f}_{1, i}=\int_{0}^{1} \int_{0}^{1} \widetilde{\Omega}_{i} d Y d Z ; \bar{f}_{2, i}=\int_{0}^{1} \int_{0}^{1} \tilde{\Phi}_{i} d Y d Z
\end{aligned}
$$


These coefficients can be analytically determined, computed at once, and then stored in arrays that during the execution of the main algorithm should be recalled, thus reducing the computational cost of the numerical solution of the transformed system.

The average concentrations of the species along the microreactor can then be calculated from the following equations:

$C_{r, A v}(X)=\frac{\sum_{i=1}^{\infty}\left[\int_{0}^{1} \int_{0}^{1} U_{T G}(Y, Z) \widetilde{\Omega}_{i}(Y, Z) d Y d Z\right] \bar{C}_{r_{i}}(X)}{\int_{0}^{1} \int_{0}^{1} U_{T G}(Y, Z) d Y d Z}$,

where $r=T G, D G, M G, G L, B$

$C_{A, A v}(X)=\frac{\sum_{i=1}^{\infty}\left[\int_{0}^{1} \int_{0}^{1} U_{T G}(Y, Z) \tilde{\Phi}_{i}(Y, Z) d Y d Z\right] \bar{C}_{A h_{i}}(X)}{\int_{0}^{1} \int_{0}^{1} U_{T G}(Y, Z) d Y d Z}+C_{A e q}$

\section{Appendix C. Solution methodology for the concentration field with eigenvalue problem accounting for the velocity profile}

Now, eigenvalue problems with variable coefficients will be proposed aiming to accelerate the convergence behavior of the solutions by building eigenfunctions with further information on the physical phenomenon. Table $\mathrm{C} 1$ shows the new formulation for the eigenvalue problems containing the information of the velocity profile.

This new formulation for the eigenvalue problems does not have a closed form analytical solution, but it can be solved by the GITT approach itself. For this purpose, simpler auxiliary problems should be proposed in order to support the representation of the eigenfunctions in terms of simpler auxiliary eigenfunction expansions. In this case, the same eigenvalue problems defined in Table B1 can be considered.

The orthogonality property of the eigenfunctions is used to define the transform and inverse formulae for the original eigenfunctions $\Gamma$ and $\Psi$ presented in Table C1, respectively, by Eqs. $(\mathrm{C} 1, \mathrm{C} 2)$ :

$\bar{\Gamma}_{i l}=\int_{0}^{1} \int_{0}^{1} \tilde{\Phi}_{l}(Y, Z) \Gamma_{i}(Y, Z) d Y d Z$

$\bar{\Psi}_{i l}=\int_{0}^{1} \int_{0}^{1} \widetilde{\Omega}_{l}(Y, Z) \Psi_{i}(Y, Z) d Y d Z$

$\Gamma_{i}(Y, Z)=\sum_{l=1}^{\infty} \tilde{\Phi}_{l}(Y, Z) \bar{\Gamma}_{i l}$

$\Psi_{i}(Y, Z)=\sum_{l=1}^{\infty} \tilde{\Omega}_{l}(Y, Z) \bar{\Psi}_{i l}$

where $\bar{\Gamma}_{i l}$ and $\bar{\Psi}_{i l}$ are eigenvectors that will be obtained from the integral transform solution of the eigenvalue problems defined in Table C1.

The integral transformation for the eigenvalue problems presented in Table $\mathrm{C} 1$ is accomplished by multiplying the respective normalized auxiliary eigenfunction given in Eqs. (B1.a,b) for each eigenvalue problem, followed by integration over the domain $[0,1]$ in the $Y$ and $Z$ directions, and then substituting the inverse formulae for the original eigenfunctions, $\Gamma$ and $\Psi[33,37]$. After performing the integral transformation procedure, the following algebraic systems for calculating the eigenvalues and corresponding eigenvectors are obtained:

$\left[-\gamma_{l}^{2} \delta_{l m}+\beta_{i}^{2} O_{2, l m}\right] \bar{\Gamma}_{i m}=0$
$\left[-v_{l}^{2} \delta_{l m}+\lambda_{i}^{2} O_{1, l m}\right] \bar{\Psi}_{i m}=0$

Equations (C3) form an infinite algebraic system that can be numerically solved after a sufficiently large truncation order MT to ensure the desired accuracy for the eigenvalues $\beta_{i}$ and $\lambda_{i}$. The norms of the eigenfunctions $\Gamma_{i}(Y, Z)$ and $\Psi_{i}(Y, Z)$ are then computed, respectively, as:

$\operatorname{Norm}_{\Gamma, i}=\int_{0}^{1} \int_{0}^{1} U_{T G}(Y, Z) \Gamma_{i}^{2}(Y, Z) d Y d Z=\sum_{l=1}^{M T} \sum_{m=1}^{M T}\left(O_{2, l m} \bar{\Gamma}_{i m}\right) \bar{\Gamma}_{i l}$

$\operatorname{Norm}_{\Psi, i}=\int_{0}^{1} \int_{0}^{1} U_{T G}(Y, Z) \Psi_{i}^{2}(Y, Z) d Y d Z=\sum_{l=1}^{M T} \sum_{m=1}^{M T}\left(O_{1, l m} \bar{\Psi}_{i m}\right) \bar{\Psi}_{i l}$

After the eigenvalue problem solution, the transform and inverse formulae for the concentration of all species involved in the transesterification reaction can be defined, respectively, as:

$$
\begin{aligned}
& \bar{C}_{A h_{i}}(X)=\int_{0}^{1} \int_{0}^{1} U_{T G}(Y, Z) \tilde{\Gamma}_{i}(Y, Z) C_{A h}(X, Y, Z) d Y d Z \\
& \bar{C}_{r_{i}}(X)=\int_{0}^{1} \int_{0}^{1} U_{T G}(Y, Z) \tilde{\Psi}_{i}(Y, Z) C_{r}(X, Y, Z) d Y d Z \\
& C_{A h}(X, Y, Z)=\sum_{i=1}^{\infty} \tilde{\Gamma}_{i}(Y, Z) \bar{C}_{A h_{i}}(X) ; \\
& C_{r}(X, Y, Z)=\sum_{i=1}^{\infty} \tilde{\Psi}_{i}(Y, Z) \bar{C}_{r_{i}}(X)
\end{aligned}
$$

with $r=T G, D G, M G, B, G$, where $\tilde{\Gamma}_{i}(Y, Z)$ and $\tilde{\Psi}_{i}(Y, Z)$ are the normalized eigenfunctions defined as:

$\tilde{\Gamma}_{i}(Y, Z)=\frac{\Gamma_{i}(Y, Z)}{\sqrt{\text { Norm }_{\Gamma, i}}} ; \tilde{\Psi}_{i}(Y, Z)=\frac{\Psi_{i}(Y, Z)}{\sqrt{\text { Norm }_{\Psi, i}}}$

The integral transformation of the filtered partial differential equations system can be carried out operating with the integral operator of the respective eigenfunctions for each species over the domain [0,1], in the $Y$ and $Z$ directions, then using the boundary conditions together with the second Green's formula. The transformed boundary conditions in the $X$ direction are obtained by transforming Eq. (6.b-d) with the aid of the weighting function of Eqs. (C5.a,b). Finally, it is obtained the following system of transformed equations: 
$\frac{d \bar{C}_{r_{i}}}{d X}=\frac{1}{P e_{r}} \sum_{j=1}^{\infty} \sum_{l=1}^{\infty} O_{1, i j l} \frac{d^{2} \bar{C}_{r_{j}}}{d X^{2}}-\xi_{r} \lambda_{i}^{2} \bar{C}_{r_{i}}+\varsigma \bar{G}_{r, i j k l m n}$,

where $r=T G, D G, M G, B, G L$

$\frac{d \bar{C}_{A h_{i}}}{d X}=\frac{1}{P e_{A h}} \sum_{j=1}^{\infty} \sum_{l=1}^{\infty} O_{2, i j l} \frac{d^{2} \bar{C}_{A h_{j}}}{d X^{2}}-\xi_{A h} \beta_{i}^{2} \bar{C}_{A h_{i}}+\varsigma \bar{G}_{A h, i j k l m n}$

$\bar{C}_{T G_{i}}(0)=\sum_{l=1}^{\infty} \bar{f}_{1, i l} ;$

$\bar{C}_{A h_{i}}(0)=\sum_{l=1}^{\infty} \bar{f}_{2, i l} ; \bar{C}_{r_{i}}(0)=0$,

(C8.c-e)

where $r=D G, M G, B, G L$

$\left.\frac{d \bar{C}_{r_{i}}}{d X}\right|_{X=1}=0$, where $r=T G, A, D G, M G, B, G L$

where, the transformed kinetic expressions $\bar{G}$ are presented in Table $\mathrm{C} 2$.

The various coefficients that appear in the integral transformation procedure are defined as:

$$
C_{r, A v}=\frac{\left[\sum_{i=1}^{\infty} \sum_{l=1}^{\infty} \int_{0}^{1} \int_{0}^{1} U_{T G}(Y, Z) \widetilde{\Omega}_{l}(Y, Z) d Y d Z \bar{\Psi}_{i l}\right] \bar{C}_{r_{i}}(X)}{\int_{0}^{1} \int_{0}^{1} U_{T G}(Y, Z) d Y d Z},
$$

where $r=T G, D G, M G, G L, B$

$$
C_{A, A v}=\frac{\left[\sum_{i=1}^{\infty} \sum_{l=1}^{\infty} \int_{0}^{1} \int_{0}^{1} U_{T G}(Y, Z) \tilde{\Phi}_{l}(Y, Z) d Y d Z \bar{\Gamma}_{i l}\right] \bar{C}_{A h_{i}}(X)}{\int_{0}^{1} \int_{0}^{1} U_{T G}(Y, Z) d Y d Z}
$$

$$
+C_{\text {Aeq }}
$$

$$
\begin{aligned}
& O_{1, j l}=\bar{\Psi}_{i l} \bar{\Psi}_{j l}, \quad O_{2, i j l}=\bar{\Gamma}_{i l} \bar{T}_{j l} ; \quad O_{3, j k l m n}=\int_{0}^{1} \int_{0}^{1} \tilde{\Omega}_{l} \tilde{\Omega}_{m} \tilde{\Phi}_{n} d Y d Z \bar{\Psi}_{i l} \bar{\Psi}_{j m} \bar{T}_{k n} ; \\
& O_{4, j k l m n}=\int_{0}^{1} \int_{0}^{1} \tilde{\Omega}_{l} \tilde{\Omega}_{m} \tilde{\Omega}_{n} d Y d Z \bar{\Psi}_{i l} \bar{\Psi}_{j m} \bar{\Psi}_{k n} ; \quad O_{5, i j k l m n}=\int_{0}^{1} \int_{0}^{1} \tilde{\Phi}_{l} \tilde{\Omega}_{m} \tilde{\Phi}_{n} d Y d Z \bar{\Gamma}_{i l} \bar{\Psi}_{j m} \bar{\Gamma}_{k n} ; \\
& O_{6, i j k l m n}=\int_{0}^{1} \int_{0}^{1} \tilde{\Phi}_{l} \tilde{\Omega}_{m} \tilde{\Omega}_{n} d Y d Z \bar{\Gamma}_{i l} \bar{\Psi}_{j m} \bar{\Psi}_{k n} ; \quad O_{7, i j l m}=\int_{0}^{1} \int_{0}^{1} \tilde{\Phi}_{l} \tilde{\Omega}_{m} d Y d Z \bar{\Gamma}_{i l} \bar{\Psi}_{j m} ; \\
& \bar{f}_{1, i l}=\int_{0}^{1} \int_{0}^{1} U_{T G} \tilde{\Omega}_{l} d Y d Z \bar{\Psi}_{i l} ; \bar{f}_{2, i l}=-F_{A o} \int_{0}^{1} \int_{0}^{1} U_{T G} \tilde{\Phi}_{l} d Y d Z \bar{T}_{i l}
\end{aligned}
$$

Again, these coefficients can be analytically determined and computed only once, and then stored in arrays that during the execution of the main algorithm can be recalled, reducing the computational cost on the numerical solution of the transformed system.

The average concentration of the species along the microreactor can then be calculated from the following equations:
Table B1

Eigenvalue problems with constant coefficients.

\begin{tabular}{ll}
\hline For the alcohol species & For other species \\
\hline$\frac{\partial^{2} \Phi_{i}}{\partial Y^{2}}+\omega \frac{\partial^{2} \Phi_{i}}{\partial Z^{2}}+\gamma_{i}^{2} \Phi_{i}(Y, Z)=0$ & $\frac{\partial^{2} \Omega_{i}}{\partial Y^{2}}+\omega \frac{\partial^{2} \Omega_{i}}{\partial Z^{2}}+v_{i}^{2} \Omega_{i}(Y, Z)=0$ \\
$\left.\frac{\partial \Phi_{i}}{\partial Y}\right|_{Y=0}=0 ; \Phi_{i}(1, Z)=0$ & $\left.\frac{\partial \Omega_{i}}{\partial Y}\right|_{Y=0}=0 ;\left.\frac{\partial \Omega_{i}}{\partial Y}\right|_{Y=1}=0$ \\
$\left.\frac{\partial \Phi_{i}}{\partial Z}\right|_{Z=0}=0 ;\left.\frac{\partial \Phi_{i}}{\partial Z}\right|_{Z=1}=0$ & $\left.\frac{\partial \Omega_{i}}{\partial Z}\right|_{Z=0}=0 ;\left.\frac{\partial \Omega_{i}}{\partial Z}\right|_{Z=1}=0$ \\
\hline
\end{tabular}


Table B2

Auxiliary eigenvalue problems to solve the eigenvalue problems for $\Phi(\mathrm{Y}, \mathrm{Z})$ and $\Omega(\mathrm{Y}, \mathrm{Z})$.

\begin{tabular}{|c|c|c|}
\hline \multicolumn{3}{|c|}{ For the original eigenfunction $\Phi(Y, Z)$} \\
\hline & Direction $Y$ & Direction $Z$ \\
\hline Eigenvalue problems & $\frac{d^{2} \phi_{1, m}}{d Y^{2}}+\gamma_{1, m}^{2} \phi_{1, m}(Y)=0$ & $\omega \frac{d^{2} \phi_{2, n}}{d Z^{2}}+\gamma_{2, n}^{2} \phi_{2, n}(Z)=0$ \\
\hline & $\left.\frac{d \phi_{1, m}}{d Y}\right|_{Y=0}=0 ; \quad \phi_{1, m}(1)=0$ & $\left.\frac{d \phi_{2, n}}{d Z}\right|_{Z=0}=0 ;\left.\quad \frac{d \phi_{2, n}}{d Z}\right|_{Z=1}=0$ \\
\hline Normalized eigenfunctions & $\tilde{\phi}_{1, m}(Y)=\sqrt{2} \cos \left(\gamma_{1, m} Y\right)$ & $\tilde{\phi}_{2, n}(Z)=\left\{\begin{array}{c}1, \quad \text { if } \quad \gamma_{2, n}=0 \\
\sqrt{2} \cos \left(\frac{\gamma_{2, n}}{\sqrt{\omega}} Z\right), \quad \text { if } \gamma_{2, n} \neq 0\end{array}\right.$ \\
\hline Eigenvalues & $\gamma_{1, m}=\frac{(2 m-1) \pi}{2}, \quad m=1,2,3, \ldots$ & $\gamma_{2, n}=\sqrt{\omega}(n-1) \pi, \quad n=1,2,3, \ldots$ \\
\hline \multicolumn{3}{|c|}{ For the original eigenfunction $\Omega(\mathrm{Y}, \mathrm{Z})$} \\
\hline & Direction $Y$ & Direction $Z$ \\
\hline Eigenvalue problems & $\frac{d^{2} \varphi_{1, m}}{d Y^{2}}+v_{1, m}^{2} \varphi_{1, m}(Y)=0$ & $\omega \frac{d^{2} \varphi_{2, n}}{d Y^{2}}+v_{2, n}^{2} \varphi_{2, n}(Y)=0$ \\
\hline & $\left.\frac{d \varphi_{1, m}}{d Y}\right|_{Y=0}=0 ;\left.\quad \frac{d \varphi_{1, m}}{d Y}\right|_{Y=1}=0$ & $\left.\frac{d \varphi_{2, n}}{d Y}\right|_{Y=0}=0 ;\left.\frac{d \varphi_{2, n}}{d Y}\right|_{Y=1}=0$ \\
\hline Normalized eigenfunctions & $\tilde{\varphi}_{1, m}(Y)=\left\{\begin{array}{c}1, \quad \text { if } v_{1, m}=0 \\
\sqrt{2} \cos \left(v_{1, m} Y\right), \quad \text { if } v_{1, m} \neq 0\end{array}\right.$ & $\tilde{\varphi}_{2, n}(Z)=\left\{\begin{array}{c}1, \quad \text { if } \quad v_{2, n}=0 \\
\sqrt{2} \cos \left(\frac{v_{2, n}}{\sqrt{\omega}} Z\right), \quad \text { if } \quad v_{2, n} \neq 0\end{array}\right.$ \\
\hline Eigenvalues & $v_{1, m}=(m-1) \pi, \quad m=1,2,3, \ldots$ & $v_{2, n}=\sqrt{\omega}(n-1) \pi, \quad n=1,2,3, \ldots$ \\
\hline
\end{tabular}

Table B3

Transformed kinetic terms for the different species involved in the transesterification reaction in the GITT solution with eigenvalue problems of constant coefficients.

\begin{tabular}{ll}
\hline Specie $\boldsymbol{r}$ & $\overline{\boldsymbol{G}}_{\boldsymbol{r}, i j \boldsymbol{k}}$ \\
\hline$T G$ & $\sum_{k=1}^{\infty} \sum_{j=1}^{\infty}\left(-k_{1} O_{3, j i k} \bar{C}_{T G_{j}} \bar{C}_{A h_{k}}+k_{2} O_{4, i j k} \bar{C}_{D G_{j}} \bar{C}_{B_{k}}\right)-k_{1} C_{A e q} \bar{C}_{T G_{i}}$ \\
$A$ & $\sum_{k=1}^{\infty} \sum_{j=1}^{\infty}\left[O_{5, i j k}\left(-k_{1} \bar{C}_{T G_{j}}-k_{3} \bar{C}_{D G_{j}}-k_{5} \bar{C}_{M G_{j}}\right) \bar{C}_{A h_{k}}+O_{6, i j k}\left(k_{2} \bar{C}_{D G_{j}}+k_{4} \bar{C}_{M G_{j}}+k_{6} \bar{C}_{G L_{j}}\right) \bar{C}_{B_{k}}\right]$ \\
& $+C_{A e q} \sum_{j=1}^{\infty} O_{7, i j}\left(-k_{1} \bar{C}_{T G_{j}}-k_{3} \bar{C}_{D G_{j}}-k_{5} \bar{C}_{M G_{j}}\right)$ \\
& \\
& $\sum_{k=1}^{\infty} \sum_{j=1}^{\infty}\left[O_{3, i j k}\left(-k_{1} \bar{C}_{T G_{j}}-k_{3} \bar{C}_{D G_{j}}\right) \bar{C}_{A h_{k}}+O_{4, i j k}\left(-k_{2} \bar{C}_{D G_{j}}+k_{4} \bar{C}_{M G_{j}}\right) \bar{C}_{B_{k}}\right]$ \\
& $+C_{A e q}\left(-k_{1} \bar{C}_{T G_{i}}-k_{3} \bar{C}_{D G_{i}}\right)$ \\
& $\sum_{k=1}^{\infty} \sum_{j=1}^{\infty}\left[O_{3, i j k}\left(k_{3} \bar{C}_{D G_{j}}-k_{5} \bar{C}_{M G_{j}}\right) \bar{C}_{A h_{k}}+O_{4, j i k}\left(-k_{4} \bar{C}_{M G_{j}}+k_{6} \bar{C}_{G L_{j}}\right) \bar{C}_{B_{k}}\right]$ \\
& $+C_{A e q}\left(k_{3} \bar{C}_{D G_{i}}-k_{5} \bar{C}_{M G_{i}}\right)$ \\
& $\sum_{k=1}^{\infty} \sum_{j=1}^{\infty}\left(k_{5} O_{3, i j k} \bar{C}_{M G_{j}} \bar{C}_{A h_{k}}-k_{6} O_{4, i j k} \bar{C}_{G L_{j}} \bar{C}_{B_{k}}\right)+k_{5} C_{A e q} \bar{C}_{M G_{j}}$ \\
& $\sum_{k=1}^{\infty} \sum_{j=1}^{\infty}\left[O_{3, i j k}\left(k_{1} \bar{C}_{T G_{j}}+k_{3} \bar{C}_{D G_{j}}+k_{5} \bar{C}_{M G_{j}}\right) \bar{C}_{A h_{k}}+O_{4, i j k}\left(-k_{2} \bar{C}_{D G_{j}}+k_{4} \bar{C}_{M G_{j}}-k_{6} \bar{C}_{G L_{j}}\right) \bar{C}_{B_{k}}\right]$ \\
& $+C_{A e q}\left(k_{1} \bar{C}_{T G_{i}}+k_{3} \bar{C}_{D G_{i}}+k_{5} \bar{C}_{M G_{i}}\right)$ \\
\hline &
\end{tabular}

Table C1

Eigenvalue problems with variable coefficients.

\begin{tabular}{ll}
\hline For the alcohol species & For other species \\
\hline$\frac{\partial^{2} \Gamma_{i}}{\partial Y^{2}}+\omega \frac{\partial^{2} \Gamma_{i}}{\partial Z^{2}}+\beta_{i}^{2} U_{T G}(Y, Z) \Gamma_{i}(Y, Z)=0$ & $\frac{\partial^{2} \Psi_{i}}{\partial Y^{2}}+\omega \frac{\partial^{2} \Psi_{i}}{\partial Z^{2}}+\lambda_{i}^{2} U_{T G}(Y, Z) \Psi_{i}(Y, Z)=0$ \\
$\left.\frac{\partial \Gamma_{i}}{\partial Y}\right|_{Y=0}=0 ; \quad \Gamma_{i}(1, Z)=0$ & $\left.\frac{\partial \Psi_{i}}{\partial Y}\right|_{Y=0}=0 ;\left.\quad \frac{\partial \Psi_{i}}{\partial Y}\right|_{Y=1}=0$ \\
$\left.\frac{\partial \Gamma_{i}}{\partial Z}\right|_{Z=0}=0 ;\left.\quad \frac{\partial \Gamma_{i}}{\partial Z}\right|_{Z=1}=0$ & $\left.\frac{\partial \Psi_{i}}{\partial Z}\right|_{Z=0}=0 ;\left.\frac{\partial \Psi_{i}}{\partial Z}\right|_{Z=1}=0$ \\
\hline
\end{tabular}


Table C2

Transformed kinetic terms for the different species involved in the transesterification reaction in the GITT solution with variable coefficients eigenvalue problems.

\begin{tabular}{|c|c|}
\hline Specie $\boldsymbol{r}$ & $\bar{G}_{r, \boldsymbol{i j k l m n}}$ \\
\hline$T G$ & $\sum_{j=1}^{\infty} \sum_{k=1}^{\infty} \sum_{l=1}^{\infty} \sum_{m=1}^{\infty} \sum_{n=1}^{\infty}\left(-k_{1} O_{3, i j k l m n} \bar{C}_{T G_{j}} \bar{C}_{A h_{k}}+k_{2} O_{4, i j k l m n} \bar{C}_{D G_{j}} \bar{C}_{B_{k}}\right)-k_{1} C_{A e q} \sum_{j=1}^{\infty} \sum_{l=1}^{\infty} O_{1, i j l} \bar{C}_{T G_{j}}$ \\
\hline$A$ & $\begin{array}{l}\sum_{j=1}^{\infty} \sum_{k=1}^{\infty} \sum_{l=1}^{\infty} \sum_{m=1}^{\infty} \sum_{n=1}^{\infty}\left[O_{5, i j k l m n}\left(-k_{1} \bar{C}_{T G_{j}}-k_{3} \bar{C}_{D G_{j}}-k_{5} \bar{C}_{M G_{j}}\right) \bar{C}_{A h_{k}}+O_{6, i j k l m n}\left(k_{2} \bar{C}_{D G_{j}}+k_{4} \bar{C}_{M G_{j}}+k_{6} \bar{C}_{G L_{j}}\right) \bar{C}_{B_{k}}\right. \\
+C_{A e q} \sum_{j=1}^{\infty} \sum_{l=1}^{\infty} \sum_{m=1}^{\infty} O_{7, i j l m}\left(-k_{1} \bar{C}_{T G_{j}}-k_{3} \bar{C}_{D G_{j}}-k_{5} \bar{C}_{M G_{j}}\right)\end{array}$ \\
\hline$D G$ & $\begin{array}{l}\sum_{j=1}^{\infty} \sum_{k=1}^{\infty} \sum_{l=1}^{\infty} \sum_{m=1}^{\infty} \sum_{n=1}^{\infty}\left[O_{3, i j k l m n}\left(-k_{1} \bar{C}_{T G_{j}}-k_{3} \bar{C}_{D G_{j}}\right) \bar{C}_{A h_{k}}+O_{4, j i k l m n}\left(-k_{2} \bar{C}_{D G_{j}}+k_{4} \bar{C}_{M G_{j}}\right) \bar{C}_{B_{k}}\right] \\
+C_{\text {Aeq }} \sum_{j=1}^{\infty} \sum_{l=1}^{\infty} O_{1, j j l}\left(k_{1} \bar{C}_{T G_{j}}-k_{3} \bar{C}_{D G_{j}}\right)\end{array}$ \\
\hline$M G$ & $\begin{array}{l}\sum_{j=1}^{\infty} \sum_{k=1}^{\infty} \sum_{l=1}^{\infty} \sum_{m=1}^{\infty} \sum_{n=1}^{\infty}\left[O_{3, i j k l m n}\left(k_{3} \bar{C}_{D G_{j}}-k_{5} \bar{C}_{M G_{j}}\right) \bar{C}_{A h_{k}}+O_{4, j i k l m n}\left(-k_{4} \bar{C}_{M G_{j}}+k_{6} \bar{C}_{G L_{j}}\right) \bar{C}_{B_{k}}\right] \\
+C_{A e q} \sum_{m=1}^{\infty} \sum_{l=1}^{\infty} O_{7, i j l}\left(k_{3} \bar{C}_{D G_{j}}-k_{5} \bar{C}_{M G_{j}}\right)\end{array}$ \\
\hline$G L$ & $\sum_{j=1}^{\infty} \sum_{k=1}^{\infty} \sum_{l=1}^{\infty} \sum_{m=1}^{\infty} \sum_{n=1}^{\infty}\left(k_{5} O_{3, i j k l m n} \bar{C}_{M G_{j}} \bar{C}_{A h_{k}}-k_{6} O_{4, i j k l m n} \bar{C}_{G L_{j}} \bar{C}_{B_{k}}\right)+k_{5} C_{A e q} \sum_{j=1}^{\infty} \sum_{l=1}^{\infty} O_{1, i j l} \bar{C}_{M G_{j}}$ \\
\hline$B$ & $\begin{array}{l}\sum_{j=1}^{\infty} \sum_{k=1}^{\infty} \sum_{l=1}^{\infty} \sum_{m=1}^{\infty} \sum_{n=1}^{\infty}\left[O_{3, i j k l m n}\left(k_{1} \bar{C}_{T G_{j}}+k_{3} \bar{C}_{D G_{j}}+k_{5} \bar{C}_{M G_{j}}\right) \bar{C}_{A h_{k}}-O_{4, i j k l m n}\left(k_{2} \bar{C}_{D G_{j}}+k_{4} \bar{C}_{M G_{j}}+k_{6} \bar{C}_{G L_{j}}\right) \bar{C}_{B_{k}}\right] \\
+C_{A e q} \sum_{j=1}^{\infty} \sum_{l=1}^{\infty} O_{1, i j l}\left(k_{1} \bar{C}_{T G_{j}}-k_{3} \bar{C}_{D G_{j}}+k_{5} \bar{C}_{M G_{j}}\right)\end{array}$ \\
\hline
\end{tabular}

\section{References}

[1] Anastopoulos G, Zannikou Y, Stournas S, Kalligeros S. Transesterification of vegetable oils with ethanol an characterization of the key fuel properties of ethyl esters. Energies 2009;2:362-76.

[2] Xie T, Zhang L, Xu N. Biodiesel synthesis in microreactors. Green Process Synth 2012; 1:61-70.

[3] Freedman B, Butterfield RO, Pryde EH. Transesterificatin kinetics of soybean oil. J Am Oil Chemist's Soc 1986;63(n. 10):1375-80.

[4] Noureddini H, Zhu D. Kinetics of transesterification of soybean oil. J Am Oil Chemists' Soc 1997;74(11):1457-63.

[5] Chiu C-W, Goff MJ, Suppes GJ. Distribution of methanol and catalysts between biodiesel and glycerin phases. AIChE J 2005;51(n. 4):1274-8.

[6] Likozar B, Levec J. Transesterification of canola, palm, peanut, soybean and sunflower oil with methanol, ethanol, isopropanol, butanol and tert-butanol to biodiesel: modelling of chemical equilibrium, reaction kinetics and mass transfer based on fatty acid composition. Appl Energy 2014;123:108-20.

[7] Vicente G, Coteron A, Martinez M, Aracil J. Integrated biodiesel production: a comparison of different homogeneous catalyst systems. Bioresour Technol 2004:92:297-305.

[8] Charoenwat R, Dennis BH. Transesterification of vegetable oils with a continuous flow capillary reactor. In: Proceedings of the ASME 2009-early career technical conference; 2009. Arlington, Texas, USA.

[9] Hessel V, Hardt S, Löwe H. Micro chemical process engineering. Weinheim: Wiley-VCH; 2004

[10] Hessel V, Löb P, Löwe H, Meudt A, Pennemann H, Scherer S, et al. Chemica micro process engineering in the field of organic synthesis. Handouts of the API Seminar. 2004. ISPE Meeting, May 13-14, 2004, Brussels, Belgium.

[11] Hessel V, Löwe H, Muller A, Kolb G. Chemical micro process engineering: processing and plants. Weinheim: Wiley-VCH; 2005.

[12] Martinez EL, Martins PF, Jardini AL, Gutierrez-Rivera L, Maciel Filho R. Continuous synthesis and in situ monitoring of biodiesel production in different microfluidic devices. Industrial Eng Chem Res 2012:51:10755-67.

[13] Al-Dhubabian AA. Production of biodiesel from soybean oil in a micro scale reactor. Corvallis, OR, USA: Oregon State University; 2005. M.Sc. thesis.

[14] Ozkan L, Backx T, Van Gerven T, Stankiewicz AI. Towards Perfect Reactors: gaining full control of chemical transformations at molecular level. Chem Eng Process 2012;51:109-16.

[15] Nikacevic NM, Huesman AEM, Van den Hof PMJ, Stankiewicz AI. Opportunities and challenges for process control in process intensification. Chem Eng Process Process Intensif 2012;52:1-15.

[16] Sturm GSJ, Verweij MD, Stankiewicz AI, Stefanidis GD. Microwaves and microreactors: design challenges and remedies, vol. 243; 2014. p. 147-58.

[17] Malengier B, Pushpavanam S, D'haeyer S. Optimizing performance of liquid- liquid extraction in stratified flow in micro-channels. J Micromechanics Microengineering 2011;21:115030-42.

[18] Guan G, Kusakabe K, Moriyama K, Sakurai N. Transesterification of sunflower oil with methanol in a microtube reactors. Ind Eng Chem Res 2009;48: 1357-63.

[19] Guan G, Teshima M, Sato C, Son SM, Irfan MF, Kusakabe K. Two-phase flow behavior in microtube reactors during biodiesel production from waste cooking oil. AIChE J 2010;56(n. 5):1383-90.

[20] Richard R, Thiebaud-Roux S, Prat L. Modelling the kinetics of transesterification reaction of sunflower oil with ethanol in microreactors. Chem Eng Sci 2013;87:258-69.

[21] Cheng L-H, Yen S-Y, Chen Z-S, Chen J. Modeling and simulation of biodiesel production using a membrane reactor integrated with a prereactor. Chem Eng Sci 2012;69:81-92.

[22] Billo RE, Oliver CR, Charoenwat R, Dennis BH, Wilson PA, Priest JW, et al. A cellular manufacturing process for a full-scale biodiesel microreactor. J Manuf Syst 2015;1:409-16.

[23] Hasebe S. Design and operation of micro-chemical plants - bridging the gap between nano, micro and macro technologies. Comput Aided Chem Eng 2004;29:57-64.

[24] Kothare MV. Dynamics and control of integrated microchemical systems with application to micro-scale fuel processing. Comput Chem Eng 2006;30: $1725-34$.

[25] De Boer K, Bahri PA. Investigation of liquid-liquid two phase flow in biodiesel production. In: Proceedings of the seventh international conference on CFD in the minerals and process industries; 2009. Melbourne, Australia.

[26] de Boer K, Moheimani NR, Borowitzka MA, Bahri PA. Extraction and conversion pathways for microalgae to biodiesel: a review focused on energy consumption. J Appl Phycol 2012;24:1681-98.

[27] Santacesaria E, Di Serio M, Tesser R, Turco R, Tortorelli M, Russo V. Biodiesel process intensification in a very simple microchannel device. Chem Eng Process 2012;52:47-54.

[28] Santacesaria E, Di Serio M, Tesser R, Tortorelli M, Turco R, Russo V. A simple device to test biodiesel process intensification. Chem Eng Process Process Intensif 2011:50:1085-94.

[29] Santacesaria E, Vicente GM, Di Serio M, Tesser R. Main technologies in biodiesel production: state of the art and future challenges. Catal Today 2012:195:2-13.

[30] Vicente G, Coteron A, Martinez M, Aracil J. Application of the factorial design of experiments and response surface methodology to optimize biodiesel production. Industrial Crops Prod 1998;8:29-35.

[31] Cotta RM. Hybrid numerical-analytical approach to nonlinear diffusion problems. Num Heat Transf part B- Fundam 1990;127:217-26.

[32] Serfaty R, Cotta RM. Integral transform solutions of diffusion problems with 
nonlinear equation coefficients. Int Comm Heat Mass Transf 1990;17:851-64.

[33] Cotta RM. Integral transforms in computational heat and fluid flow. Boca Raton: CRC Press; 1993.

[34] Cotta RM. Benchmark results in computational heat and fluid flow: - the integral transform method, (Invited Paper). Int J Heat Mass Transf 1994;37: 381-94.

[35] Cotta RM, Mikhailov MD. Heat conduction: lumped analysis. Integral transforms. Symbolic computation. Chichester, UK: Wiley-Interscience; 1997.

[36] Cotta RM, Mikhailov MD. Hybrid methods and symbolic computations. In: Minkowycz WJ, Sparrow EM, Murthy JY, editors. Handbook of numerical heat transfer. second ed. New York: John Wiley; 2006. p. 493-522 [Chapter 16].

[37] Cotta RM, Knupp DC, Naveira-Cotta CP. Analytical heat and fluid flow in microchannels and microsystems, Mechanical engineering series. Springer International Publishing; 2015.

[38] Ribeiro NFP, Machado NT, Macedo EN, Quaresma JNN, Lima JA. Integral transform approach in chemically reacting non-Newtonian flows. Int Comm Heat Mass Trannsfer 2002;29:601-10.

[39] Pontes PC, Naveira-Cotta CP, Quaresma JNN. Mass transfer simulation of biodiesel synthesis in microreactors. Comput Chem Eng 2016;93:36-51.

[40] Pontes PC, Naveira-Cotta CP, Macedo EN, Quaresma JNN. Integral transform analysis of three-dimensional mass transfer in the transesterification process in microreactors, Proc.. of the $7^{\text {th }}$ international symposium on advances in computational heat transfer. 2015. CHT-15 ICHMT, Piscataway, NJ, May 25-29.

[41] Pontes PC, Naveira-Cotta CP. Inverse problem analysis for identification of reaction kinetics constants im microreactors for biodiesel synthesis. In: Proc.. of the $7^{\text {th }}$ european thermal-sciences conference; 2016. EUROTHERM, Krakow, Poland, Jun 19-23.

[42] Pontes PC, Oliveira KB, Macêdo EN, Quaresma JNN. Modeling and simulation of uphill transport of a diluted solute in separation processes using supported liquid membranes: an integral transform solution. In: Proc.. of the VII Congresso Nacional de Engenharia Mecânica; 2012. CONEM2012-1229, São Luis, MA, Brasil, Jul 31 to Aug 03.

[43] Mikhailov MD, Cotta RM. Mixed symbolic-numerical computation of convective heat transfer with slip flow in microchannels. Int Comm Heat Mass Transf 2005;32:341-8.

[44] Cotta RM, Castellões FV. Analysis of transient and periodic convection in microchannels via integral transforms. Prog Comput fluid Dyn 2006;6.

[45] Castellões FV, Cardoso CR, Couto P, Cotta RM. Transient analysis of slip flow and heat transfer in microchannels. Heat Transf Eng 2007;28:549-58.

[46] Naveira-Cotta CP, Cotta RM, Orlande HRB, Fudym O. Inverse analysis of forced convection in micro-channels with slip flow via integral transforms and Bayesian inference. Int J Therm Sci 2010;49:879-88.

[47] Knupp DC, Naveira-Cotta CP, Cotta RM. Theoretical-experimental analysis of conjugated heat transfer in nanocomposite heat spreaders with multiple micro-channels. Int J Heat Mass Transf 2014;74:306-18.

[48] Knupp DC, Cotta RM, Naveira-Cotta CP, Kakaç S. Transient conjugated heat transfer in microchannels: integral tranforms with single domain formulation. Int J Therm Sci 2015;88:248-57.

[49] Mikhailov MD, Özisik MN. Unified analysis and solutions of heat and mass diffusion. New York: John Wiley \& Sons; 1984.

[50] Özisik MN. Heat conduction. New York: John Wiley \& Sons; 1993.

51] Wolfram S. The Mathematica book. Wolfram Media/Cambridge; 2005.

[52] Naveira-Cotta CP, Cotta RM, Orlande HRB, Fudym O. Eigenfunction expansions for transient diffusion in heterogeneous media. Int J Heat Mass Transf 2009;52:5029-39.

[53] Levenspiel O. Chemical reaction engineering. 3 ed. New York: John Wiley \& Sons; 1999. 